Research Article

\title{
On Comparative Analysis for the Black-Scholes Model in the Generalized Fractional Derivatives Sense via Jafari Transform
}

\author{
Saima Rashid, ${ }^{1}$ Sobia Sultana, ${ }^{2}$ Rehana Ashraf, ${ }^{3}$ and Mohammed K. A. Kaabar ${ }^{4,5}$ \\ ${ }^{1}$ Department of Mathematics, Government College University, Faisalabad 38000, Pakistan \\ ${ }^{2}$ Department of Mathematics, Imam Mohammad Ibn Saud Islamic University, Riyadh 12211, Saudi Arabia \\ ${ }^{3}$ Department of Mathematics, Lahore College Women University, 54000 Lahore, Pakistan \\ ${ }^{4}$ Gofa Camp, Near Gofa Industrial College and German Adebabay, Nifas Silk-Lafto, 26649 Addis Ababa, Ethiopia \\ ${ }^{5}$ Institute of Mathematical Sciences, Faculty of Science, University of Malaya, Kuala Lumpur 50603, Malaysia \\ Correspondence should be addressed to Mohammed K. A. Kaabar; mohammed.kaabar@wsu.edu
}

Received 3 October 2021; Revised 16 November 2021; Accepted 23 November 2021; Published 8 December 2021

Academic Editor: Youssri Hassan Youssri

Copyright (C) 2021 Saima Rashid et al. This is an open access article distributed under the Creative Commons Attribution License, which permits unrestricted use, distribution, and reproduction in any medium, provided the original work is properly cited.

\begin{abstract}
The Black-Scholes model is well known for determining the behavior of capital asset pricing models in the finance sector. The present article deals with the Black-Scholes model via the Caputo fractional derivative and Atangana-Baleanu fractional derivative operator in the Caputo sense, respectively. The Jafari transform is merged with the Adomian decomposition method and new iterative transform method. It is worth mentioning that the Jafari transform is the unification of several existing transforms. Besides that, the convergence and uniqueness results are carried out for the aforesaid model. In mathematical terms, the variety of equations and their solutions have been discovered and identified with various novel features of the projected model. To provide additional context for these ideas, numerous sorts of illustrations and tabulations are presented. The precision and efficacy of the proposed technique suggest its applicability for a variety of nonlinear evolutionary problems.
\end{abstract}

\section{Introduction}

Recently, the subject of fractional calculus has garnered considerable prominence. Several well-known mathematicians have contributed to this field by proposing numerous fractional operators in various texts. The conclusions of contemporary calculus are often substantially more precise than those of ancient ones. It has presented the dynamic behavior of a variety of real-world situations that take place between two integers. Additionally, fractional operators have more dimensionality than integer differential operators such as Caputo, Liouville, Hadamard, Coimbra, Davison, Riesz, Riemann and Liouville, Weyl, and Jumarie, Caputo and Fabrizio [1], Atangana and Baleanu [2], and Scherer et al. [3] are some well-known fractional derivative formulations, see [4-9]. Furthermore, the Liouville-Caputo and $\mathrm{AB}$ operators are considered to be the best fractional filters in this field of research.

Several methods that assess fractional differential equations (FDEs) for their reliability and trustworthiness are actively considered in $[10,11]$. Certain popular approximate-analytical approaches including Adomian decomposition method [12], new iterative transform method [13], homotopy perturbation method [14], Haar wavelet method [15], Padé approximation [16], reproducing kernel Hilbert space method [17], new Legendre wavelet decomposition method [18], Bäcklund transformation method [19], and Lie symmetry analysis [20].

Fischer Black and Myron Scholes developed a mathematical model for the capital asset pricing model in 1973. The revolutionary Black-Scholes model (BSM) is the foundation of modern financial theory which is remarkable to discuss contemporary economics without referencing the innovative BSM.

The purpose of this research is to obtain new solutions by employing both decomposition method and Jafari iterative transform method into a BSM. In banking and finance, the fractional formulation of BSM is represented by [16]:

$$
\mathbf{D}_{\varsigma}^{9} \mathcal{U}+\frac{\omega^{2}}{2} \mathcal{S}^{2} \frac{\partial^{2} \mathcal{u}}{\partial \mathcal{S}^{2}}+\zeta \mathcal{S} \frac{\partial \mathcal{U}}{\partial \mathcal{S}}-\zeta \mathcal{U}=0,
$$


subject to the playoff mapping

$$
\mathcal{U}(\mathcal{S}, \mathcal{T})=\max (\mathcal{S}-E, 0)
$$

where $\mathcal{U}(\mathcal{S}, \varsigma)$ represents the option's value at $\mathcal{S}$ asset values at time $\varsigma$ and $\mathscr{T}$ is the expiry period. The fundamental stock price is indicated by the letter $E$. The risk-free mortgage to expiry is represented by the variable $\zeta$. The volatility of a financial commodity is represented by the constant $\omega$. We also include the necessary hypotheses: a constant risk-free interest rate $u$, no processing fees, the ability to purchase and sell an unlimited number of stocks, and no prohibitions on speculative trading. Finally, we include European options. Furthermore, it is remarkable that $\mathcal{U}(0, \varsigma)=0$ and $\mathcal{U}(\mathcal{S}, \mathscr{T}) \approx \mathcal{S}$ as $\mathcal{S} \mapsto \infty$. The BSM in (1) can then be represented as a parabolic diffusion equation. Introducing the subsequent transformations

$$
\mathcal{S}=E \exp \left(\mathbf{w}_{1}\right), \varsigma=\mathscr{T}-\frac{2 \tau}{\omega^{2}}, \mathcal{U}=E Q\left(\mathbf{w}_{1}, \varsigma\right)
$$

then, Equation (1) reduces to

$$
\mathbf{D}_{\varsigma}^{9} Q\left(\mathbf{w}_{1}, \varsigma\right)=\frac{\partial^{2} \mathscr{Q}\left(\mathbf{w}_{1}, \varsigma\right)}{\partial \mathbf{w}_{1}^{2}}+(\eta-1) \frac{\partial \mathscr{Q}\left(\mathbf{w}_{1}, \varsigma\right)}{\partial \mathbf{w}_{1}}-\eta \mathscr{Q}\left(\mathbf{w}_{1}, \varsigma\right)
$$

subject to initial conditions

$$
Q\left(\mathbf{w}_{1}, 0\right)=\max \left(\exp \left(\mathbf{w}_{1}\right)-1,0\right)
$$

where $\eta$ signifies the equilibrium between the free relationship in inflation and stock volatility. In [20], Cen and Le introduced the generalized fractional BSM. The BSM is described this way:

$$
\begin{aligned}
\mathbf{D}_{\varsigma}^{9} \mathscr{Q}\left(\mathbf{w}_{1}, \varsigma\right)= & -0.08\left(2+\sin \mathbf{w}_{1}\right)^{2} \mathbf{w}_{1}^{2} \frac{\partial^{2} \mathscr{Q}\left(\mathbf{w}_{1}, \varsigma\right)}{\partial \mathbf{w}_{1}^{2}} \\
& -0.06 \mathbf{w}_{1} \frac{\partial \mathscr{Q}\left(\mathbf{w}_{1}, \varsigma\right)}{\partial \mathbf{w}_{1}}+0.06 \mathbb{Q}\left(\mathbf{w}_{1}, \varsigma\right)
\end{aligned}
$$

subject to initial conditions

$$
\mathcal{Q}\left(\mathbf{w}_{1}, 0\right)=\max \left(\mathbf{w}_{1}-25 \exp (-0.06), 0\right) .
$$

The fractional BSM with one commodity has been explored extensively [21, 22]. The fractional BSM is an extended variant of the classical BSM that extends the model's limitations. Meng and Wang [23] used the BSM to investigate fractional opportunity valuation. The covered call price for bank international trade in China was calculated using the fractional BSM. Their findings suggest that when it comes to evaluating the influence of the pricing system, the fractional BSM outperforms the classical BSM [24]. Fall et al. [14] estimated the Black-Scholes option pricing equations via the homotopy perturbation method. Matadi and Zondi [25] contemplated the invariant solutions of BSM via theOrnstein-Uhlenbeck process. Kumar et al. [26] dem- onstrated numerical computation of fractional BSM arising in the financial market. Yavuz and Özdemir [27] proposed a diverse approach to the European option pricing model with a new fractional operator.

Amidst Gorge Adomian's massive boost in 1980, the Adomian decomposition method introduced a well-noted terminology. It has been intensively implemented for a diverse set of nonlinear PDEs, for instance, Fisher's model [28] and Zakharov-Kuznetsov equation [29]. The ADM was determined to be significantly related to a variety of integral transforms, including Laplace, Swai, Mohand, Aboodh, and Elzaki. Very recently, Jafari [30] propounded a well-known integral transform which is known as the Jafari transform. The dominant feature of this transformation is that it has the ability to recapture several existing transformations, see Remark 8.

In 2006, Yavuz and Özdemir [27] expounded a new iterative transform method (NITM) that is intensively employed by numerous researchers due to its frequent applicability in fractional ODES and PDEs. The recursive technique tends to the exact solution if it exists via successive approximations. A small proportion of estimates can be employed for analytical reasons with a reasonable level of accuracy for particular problems. The NITM does not need some restricted hypothesis for handling nonlinearity factors. For example, in [28], the authors employed NITM to find the numerical solution of the fifth- and sixth-order nonlinear boundary value problems, Rashid et al. [29] applied NITM to obtain the solution of the fractional FornbergWhitham equation, and Jafari [30] constructed the Laplace decomposition algorithm via NITM.

Owing to the aforementioned trend, to obtain the explicit solution of the time-fractional BSM, we employ the Jafari transform decomposition technique (JDM) and Jafari iterative transform method (JITM). The Jafari transform merged the Adomian decomposition method and a new iterative method in an efficient manner to develop novel algorithmic approaches. The Jafari transform is the refinement of several existing transforms, see Remark 8. Both projected schemes yield analytical solutions in a convergent series form. Mathematical characterizations of the BSM are illustrated via the AB fractional derivative operator in the Caputo sense. Simulation and tabulation studies depict a clear picture of the proposed approaches. The analytical solution, especially for fractional PDEs, is a useful mechanism for analyzing the behaviors of solutions that are challenging to numerically solve. The analytical solution can be used to investigate macroeconomic behaviors.

This article's entire content is divided into seven parts, which are described in the following order: Section 2 summarises and presents the core concepts and terminology of the singular power law fractional derivative and nonsingular Atangana-Baleanu fractional derivative in the Caputo sense. In Section 3, two novel algorithms are developed via the new integral transform. In Section 4, convergence and uniqueness analyses are discussed and presented for the proposed model. Section 5 is the main part of the proposed work where we present a debate on the results and their 
interpretation. Finally, concluding remarks are presented in Section 7.

\section{Preliminaries}

In this section, we present some essential concepts, notions, and definitions concerning fractional derivative operators depending on power and Mittag-Leffler as a kernel, along with the detailed consequences of the Jafari transform.

Definition 1 (see [1]). The Caputo fractional derivative (CFD) is described as follows:

${ }_{0}^{c} \mathbf{D}_{\zeta}^{9}(Q(\varsigma))= \begin{cases}\frac{1}{\Gamma(r-\vartheta)} \int_{0}^{\varsigma} \frac{Q^{(r)}\left(\mathbf{w}_{1}\right)}{\left(\varsigma-\mathbf{w}_{1}\right)^{9+1-r}} d \mathbf{w}_{1}, & r-1<\vartheta<r, \\ \frac{d^{r}}{d \varsigma^{r}} \mathscr{Q}(\varsigma), & \vartheta=r .\end{cases}$

Definition 2 (see [2]). The Atangana-Baleanu fractional derivative operator in the Caputo form $(\mathrm{ABC})$ is stated as follows:

$$
{ }_{\eta_{1}}^{A B C} \mathbf{D}_{\varsigma}^{\vartheta}(\mathscr{Q}(\varsigma))=\frac{\mathbb{A}(\vartheta)}{1-\vartheta} \int_{\eta_{1}}^{\varsigma} Q^{\prime}(\varsigma) E_{\vartheta}\left[-\frac{\vartheta\left(\varsigma-\mathbf{w}_{1}\right)^{\vartheta}}{1-\vartheta}\right] d \mathbf{w}_{1}
$$

where $Q \in \mathscr{H}^{1}\left(a_{1}, a_{2}\right)$ (Sobolev space), $a_{1}<a_{2}, \vartheta \in[0,1]$ and $\mathbb{A}(\vartheta)$ signifies a normalization function as $\mathbb{A}(\vartheta)=\mathbb{A}(0)=$ $\mathbb{A}(1)=1$.

Definition 3 (see [2]). The fractional integral of the $A B C$ operator is described as follows:

$$
\begin{aligned}
\eta_{\eta_{1}} \mathscr{F}_{\varsigma}^{\vartheta}(\mathscr{Q}(\varsigma))= & \frac{1-\vartheta}{\operatorname{A}(\vartheta)} \mathscr{Q}(\varsigma) \\
& +\frac{\vartheta}{\Gamma(\vartheta) A(\vartheta)} \int_{\eta_{1}}^{\varsigma} \mathscr{Q}\left(\mathbf{w}_{1}\right)\left(\varsigma-\mathbf{w}_{1}\right)^{\vartheta-1} d \mathbf{w}_{1} .
\end{aligned}
$$

Definition 4 (see [30]). Consider an integrable mapping $\mathbb{Q}(\varsigma)$ defined on a set $\mathscr{P}$, then

$$
\mathscr{P}=\{\mathscr{Q}(\varsigma): \exists M>0, \kappa>0,|\mathbb{Q}(\varsigma)|<M \exp (\kappa \varsigma), \text { if } \varsigma \geq 0\} .
$$

Definition 5 (see [30]). Suppose the mappings $\phi(\mathfrak{g}), \psi(\mathfrak{g})$ : $\mathbb{R}^{+} \mapsto \mathbb{R}^{+}$such that $\psi(\mathfrak{g}) \neq 0 \forall \mathfrak{g} \in \mathbb{R}^{+}$. The Jafari transform of the mapping $\mathbb{Q}(\varsigma)$ presented by $\mathbf{Q}(\mathfrak{g})$ is described as

$$
\mathbb{J}\{\mathfrak{Q}(\varsigma), \mathfrak{g}\}=\mathbf{Q}(\mathfrak{I})=\phi(\mathfrak{I}) \int_{0}^{\infty} \mathscr{Q}(\varsigma) \exp (-\psi(\mathfrak{I}) \varsigma) d \varsigma
$$

Theorem 6 (see [30]) (convolution property). For Jafari transform, the subsequent holds true:

$$
\mathbb{J}\left\{\mathbb{Q}_{1} * \mathbb{Q}_{2}\right\}=\frac{1}{\phi(\mathfrak{g})} \mathbf{Q}_{1}(\mathfrak{g}) * \mathbf{Q}_{2}(\mathfrak{g})
$$

Definition 7. The Jafari transform of the CFD operator is stated as follows:

$$
\begin{aligned}
\mathbb{J}\left\{{ }_{0}^{c} \mathbf{D}_{\varsigma}^{9}(\mathscr{Q}(\varsigma)), \mathfrak{g}\right\}= & \psi^{\vartheta}(\mathfrak{I}) \mathbf{Q}(\mathfrak{I})-\phi(\mathfrak{I}) \sum_{\kappa=0}^{9-1} \psi^{9-\kappa-1}(\mathfrak{I}) \mathscr{Q}^{(\kappa)}(0), \\
& r-1<\mathfrak{\vartheta}<r, \phi, \psi>0 .
\end{aligned}
$$

Remark 8. Definition 7 leads to the following conclusions:

(1) Taking $\phi(\mathfrak{g})=1$ and $\psi(\mathfrak{g})=\mathfrak{g}$, then we acquire the Laplace transform [35]

(2) Taking $\phi(\mathfrak{g}) 1 / \mathfrak{G}$ and $\psi(\mathfrak{g})=1 / \mathfrak{g}$, then we acquire the $\alpha$-Laplace transform [36]

(3) Taking $\phi(\mathfrak{g})=1 / \mathfrak{I}$ and $\psi(\mathfrak{I})=1 / \mathfrak{g}$, then we acquire the Sumudu transform [37]

(4) Taking $\phi(\mathfrak{g})=1 / \mathfrak{g}$ and $\psi(\mathfrak{g})=1$, then we acquire the Aboodh transform [38]

(5) Taking $\phi(\mathfrak{a})=\mathfrak{g}$ and $\psi(\mathfrak{a})=\mathfrak{g}^{2}$, then we acquire the Pourreza transform $[39,40]$

(6) Taking $\phi(\mathfrak{g})=\mathfrak{g}$ and $\psi(\mathfrak{g})=1 / \mathfrak{g}$, then we acquire the Elzaki transform [41]

(7) Taking $\phi(\mathfrak{g})=\mathbf{w}_{2}$ and $\psi(\mathfrak{g})=\mathfrak{g} / \mathbf{w}_{2}$, then we acquire the natural transform [42]

(8) Taking $\phi(\mathfrak{a})=\mathfrak{g}^{2}$ and $\psi(\mathfrak{s})=\mathfrak{g}$, then we acquire the Mohand transform [43]

(9) Taking $\phi(\mathfrak{g})=1 / \mathfrak{g}^{2}$ and $\psi(\mathfrak{g})=1 / \mathfrak{g}$, then we acquire the Sawi transform [44]

(10) Taking $\phi(\mathfrak{g})=1$ and $\psi(\mathfrak{g})=1 / \mathfrak{g}$, then we get the Kamal transform [45].

(11) Taking $\phi(\mathfrak{g})=\mathfrak{g}^{\alpha}$ and $\psi(\mathfrak{g})=1 / \mathfrak{g}$, then we acquire the $G_{-}$transform $[46,47]$

Definition 9 (see [48]). The Jafari transform of the $\mathrm{ABC}$ fractional derivative operator is described as

$$
\mathbb{J}\left\{{ }_{0}^{\mathrm{ABC}} \mathbf{D}_{\zeta}^{\vartheta}(\mathbb{Q}(\varsigma)), \mathfrak{g}\right\}(\mathfrak{\vartheta})=\frac{\mathbb{A}(\mathfrak{\vartheta}) \psi^{\vartheta}(\mathfrak{I})}{\mathcal{\vartheta}+(1-\vartheta) \psi^{\vartheta}(\mathfrak{I})}\left(\mathbf{Q}(\mathfrak{s})-\frac{\phi(\mathfrak{I})}{\psi(\mathfrak{I})} \mathscr{Q}(0)\right) .
$$

Remark 10. Definition 9 leads to the following conclusions:

(1) Taking $\phi(\mathfrak{g})=1$ and $\psi(\mathfrak{g})=\mathfrak{g}$, then we acquire the Laplace transform of $\mathrm{ABC}$ fractional derivative operator $[2,49]$ 
(2) Taking $\phi(\mathfrak{g})=\mathfrak{g}$ and $\psi(\mathfrak{g})=1 / \mathfrak{g}$, then we acquire the Elzaki transform of ABC fractional derivative operator [50]

(3) Taking $\phi(\mathfrak{g})=\psi(\mathfrak{g})=1 / \mathfrak{g}$, then we get the Sumudu transform of ABC fractional derivative operator [51]

(4) Taking $\phi(\mathfrak{g})=1$ and $\psi(\mathfrak{g})=\mathfrak{g} / \mathbf{w}_{2}$, then we get the Shehu transform of $\mathrm{ABC}$ fractional derivative operator [51]

Definition 11 (see [52]). The Mittag-Leffler function for a single parameter is described as

$$
E_{\vartheta}(z)=\sum_{\kappa=0}^{\infty} \frac{z^{\kappa}}{\Gamma(\kappa \vartheta+1)}, \vartheta, z \in \mathbb{C}, \mathfrak{R}(\vartheta) \geq 0 .
$$

\section{New Semianalytical Approach for Nonlinear PDEs}

Consider the generic fractional form of PDE:

$\mathbf{D}_{\varsigma}^{9} \mathscr{Q}\left(\mathbf{w}_{1}, \varsigma\right)+\mathbb{L} Q\left(\mathbf{w}_{1}, \varsigma\right)+\mathbb{N} Q\left(\mathbf{w}_{1}, \varsigma\right)=\mathbf{F}\left(\mathbf{w}_{1}, \varsigma\right), \varsigma>0,0<\vartheta \leq 1$,

with ICs

$$
\mathscr{Q}\left(\mathbf{w}_{1}, 0\right)=\mathscr{G}\left(\mathbf{w}_{1}\right)
$$

where $\mathbf{D}_{\varsigma}^{\vartheta}=\partial^{9} \mathscr{Q}\left(\mathbf{w}_{1}, \varsigma\right) / \partial \varsigma^{9}$ symbolizes the Caputo and ABC fractional derivative of order $\vartheta \in(0,1]$ while $\mathbb{L}$ and $\mathbb{N}$ denotes the linear and nonlinear factors, respectively. Also, $\mathbf{F}\left(\mathbf{w}_{1}, \varsigma\right)$ represents the source term.

3.1. Configuration of Jafari Decomposition Method. Taking into account the Jafari transform to (17), we acquire

$$
\mathbb{J}\left[\mathbf{D}_{\varsigma}^{9} \mathscr{Q}\left(\mathbf{w}_{1}, \varsigma\right)+\overline{\mathbb{Q}} Q\left(\mathbf{w}_{1}, \varsigma\right)+\overline{\mathbb{N}} \mathscr{Q}\left(\mathbf{w}_{1}, \varsigma\right)\right]=\mathbb{J}\left[\mathbf{F}\left(\mathbf{w}_{1}, \varsigma\right)\right] .
$$

Firstly, the differentiation rule of Jafari transform with respect to CFD was applied; then, we apply the $\mathrm{ABC}$ fractional derivative operator as follows:

$$
\begin{aligned}
& \psi^{9}(\varsigma) \mathcal{U}\left(\mathbf{w}_{1}, \varsigma\right)=\phi(\varsigma) \sum_{q=0}^{n-1} \psi^{9-1-q}(\varsigma) \mathbb{Q}^{(q)}(0) \\
& +\mathbb{J}\left[\overline{\mathbb{L}} Q\left(\mathbf{w}_{1}, \varsigma\right)+\overline{\mathbb{N}} Q\left(\mathbf{w}_{1}, \varsigma\right)\right] \\
& +\mathbb{J}\left[\mathbf{F}\left(\mathbf{w}_{1}, \varsigma\right)\right] \text {, } \\
& \frac{\psi^{\vartheta}(\varsigma) \mathbb{A}(\vartheta)}{\vartheta+(1-\vartheta) \psi^{9}(\varsigma)} \mathcal{U}\left(\mathbf{w}_{1}, \varsigma\right) \\
& =\frac{\phi(\varsigma)}{\psi(\varsigma)} \frac{\psi^{9}(\varsigma) \mathbb{A}(\vartheta)}{\vartheta+(1-\vartheta) \psi^{9}(\varsigma)} Q(0) \\
& +\mathbb{J}\left[\overline{\mathbb{L}} Q\left(\mathbf{w}_{1}, \varsigma\right)+\overline{\mathbb{N}} \mathcal{Q}\left(\mathbf{w}_{1}, \varsigma\right)\right]+\mathbb{I}\left[\mathbf{F}\left(\mathbf{w}_{1}, \varsigma\right)\right] \text {. }
\end{aligned}
$$

The inverse Jafari transform of (20) and (21), respectively, yields

$$
\begin{aligned}
\mathscr{Q}\left(\mathbf{w}_{1}, \varsigma\right)= & \mathbb{J}^{-1}\left[\phi(\varsigma) \sum_{q=0}^{n-1} \psi(\varsigma)^{\vartheta-q-1} Q^{(q)}(0)+\frac{1}{\psi^{9}(\varsigma)} \mathbb{J}\left[\mathbf{F}\left(\mathbf{w}_{1}, \varsigma\right)\right]\right] \\
& -\mathbb{J}^{-1}\left[\frac{1}{\psi^{9}(\varsigma)} \mathbb{J}\left[\overline{\mathbb{Q}} \mathcal{Q}\left(\mathbf{w}_{1}, \varsigma\right)+\overline{\mathbb{N}} Q\left(\mathbf{w}_{1}, \varsigma\right)\right]\right] . \\
\mathscr{Q}\left(\mathbf{w}_{1}, \varsigma\right)= & \mathbb{J}^{-1}\left[\frac{\phi(\varsigma)}{\psi(\varsigma)} \mathscr{Q}(0)+\frac{\vartheta+(1-\vartheta) \psi^{9}(\varsigma)}{\psi^{9}(\varsigma) \mathbb{A}(\vartheta)} \mathbb{J}\left[\mathbf{F}\left(\mathbf{w}_{1}, \varsigma\right)\right]\right] \\
& -\mathbb{J}^{-1}\left[\frac{\vartheta+(1-\vartheta) \psi^{\vartheta}(\varsigma)}{\psi^{9}(\varsigma) \mathbb{A}(\vartheta)} \mathbb{J}\left[\overline{\mathbb{L} Q}\left(\mathbf{w}_{1}, \varsigma\right)+\overline{\mathbb{N}} \mathscr{Q}\left(\mathbf{w}_{1}, \varsigma\right)\right]\right] .
\end{aligned}
$$

Therefore, the Jafari decomposition method was utilized to derive the solution of (17) by satisfying the assumption that $Q\left(\mathbf{w}_{1}, \varsigma\right)$ has a solution of this equation which can be expressed as

$$
\widehat{Q}\left(\mathbf{w}_{1}, \varsigma\right)=\sum_{q=0}^{\infty} \widehat{Q}_{q}\left(\mathbf{w}_{1}, \varsigma\right)
$$

Thus, the nonlinear term $\overline{\mathbb{N}}\left(\mathbf{w}_{1}, \varsigma\right)$ can be evaluated by the Adomian decomposition method prescribed as

$$
\overline{\mathbb{N}} Q\left(\mathbf{w}_{1}, \varsigma\right)=\sum_{q=0}^{\infty} \tilde{A}_{q}\left(\widehat{Q}_{0}, \mathcal{Q}_{1}, \cdots\right), q=0,1, \cdots
$$

where

$$
\tilde{A}_{q}\left(\mathcal{Q}_{0}, \mathcal{Q}_{1}, \cdots\right)=\frac{1}{q !}\left[\frac{d^{q}}{d \vartheta^{q}} \overline{\mathbb{N}}\left(\sum_{j=0}^{\infty} \vartheta^{j} Q_{j}\right)\right]_{\vartheta=0}, q>0
$$

Inserting (23) and (24) into (26) and (27), respectively, we have

$$
\begin{gathered}
\sum_{q=0}^{\infty} \mathbb{Q}_{q}\left(\mathbf{w}_{1}, \varsigma\right)=\mathscr{G}\left(\mathbf{w}_{1}\right)+\tilde{\mathscr{G}}\left(\mathbf{w}_{1}\right) \\
-\mathbb{J}^{-1}\left[\frac{1}{\psi^{9}(\varsigma)} \mathbb{J}\left[\overline{\mathbb{L}} \mathscr{Q}\left(\mathbf{w}_{1}, \varsigma\right)+\sum_{q=0}^{\infty} \tilde{A}_{q}\right]\right] \\
\sum_{q=0}^{\infty} \mathscr{Q}_{q}\left(\mathbf{w}_{1}, \varsigma\right)=\mathscr{G}\left(\mathbf{w}_{1}\right)+\tilde{\mathscr{G}}\left(\mathbf{w}_{1}\right) \\
-\mathbb{J}^{-1}\left[\frac{\vartheta+(1-\vartheta) \psi^{9}(\varsigma)}{\mathbb{A}(\vartheta) \psi^{9}(\varsigma)} \mathbb{J}\left[\overline{\mathbb{L} Q}\left(\mathbf{w}_{1}, \varsigma\right)+\sum_{q=0}^{\infty} \tilde{A}_{q}\right]\right] .
\end{gathered}
$$

Consequently, the recursive technique for (26) and (18) are established as 


$$
\begin{gathered}
\mathscr{Q}_{0}\left(\mathbf{w}_{1}, \varsigma\right)=\mathscr{G}\left(\mathbf{w}_{1}\right)+\tilde{\mathscr{G}}\left(\mathbf{w}_{1}\right), q=0, \\
\mathscr{Q}_{q+1}\left(\mathbf{w}_{1}, \varsigma\right)=-\mathbb{J}^{-1}\left[\frac{1}{\psi^{9}(\varsigma)} \mathbb{J}\left[\overline{\mathbb{L}}\left(\mathscr{Q}_{q}\left(\mathbf{w}_{1}, \varsigma\right)\right)+\sum_{q=0}^{\infty} \tilde{A}_{q}\right]\right], q \geq 1, \\
\mathscr{Q}_{q+1}\left(\mathbf{w}_{1}, \varsigma\right)=-\mathbb{J}^{-1}\left[\frac{\vartheta+(1-\vartheta) \psi^{\vartheta}(\varsigma)}{\mathbb{A}(\vartheta) \psi^{9}(\varsigma)} \mathbb{J}\left[\overline{\mathbb{L}}\left(\mathscr{Q}_{q}\left(\mathbf{w}_{1}, \varsigma\right)\right)+\sum_{q=0}^{\infty} \tilde{A}_{q}\right]\right], q \geq 1 .
\end{gathered}
$$

3.2. Construction of Jafari Iterative Transform Method. With the aid of Jafari transform to (17) along with the IC (18), we obtain

$$
\mathbb{J}\left[\mathbf{D}_{\varsigma}^{9} \mathscr{Q}\left(\mathbf{w}_{1}, \varsigma\right)+\overline{\mathbb{Q}} Q\left(\mathbf{w}_{1}, \varsigma\right)+\overline{\mathbb{N}} Q\left(\mathbf{w}_{1}, \varsigma\right)\right]=\mathbb{I}\left[\mathbf{F}\left(\mathbf{w}_{1}, \varsigma\right)\right] .
$$

First, we apply the differentiation rule of Jafari transform for $\mathrm{CFD}$, and then, we consider for $\mathrm{ABC}$ fractional derivative operator, respectively, we get

$$
\begin{aligned}
& \mathbb{J}\left[\mathscr{Q}\left(\mathbf{w}_{1}, \varsigma\right)\right]=\frac{\phi(\varsigma)}{\psi^{9}(\varsigma)} \sum_{q=0}^{n-1} \psi^{9-1-q}(\varsigma) \mathscr{Q}^{(q)}(\psi, 0) \\
& -\frac{1}{\psi^{9}(\varsigma)} \mathbb{J}\left[\overline{\mathbb{L}} Q\left(\mathbf{w}_{1}, \varsigma\right)+\overline{\mathbb{N}} Q\left(\mathbf{w}_{1}, \varsigma\right)\right] \\
& +\frac{1}{\psi^{9}(\varsigma)} \mathbb{I}\left[\mathbf{F}\left(\mathbf{w}_{1}, \varsigma\right)\right], \\
& \mathbb{D}\left[Q\left(\mathbf{w}_{1}, \varsigma\right)\right]=\frac{\phi(\varsigma)}{\psi^{9}(\varsigma)} \sum_{q=0}^{n-1} \psi^{9-1-q}(\varsigma) Q^{(q)}(\psi, 0) \\
& -\frac{\psi^{\vartheta}(\varsigma)(1-\vartheta)+\vartheta}{\mathbb{A}(\vartheta) \psi^{\vartheta}(\varsigma)} \mathbb{J}\left[\overline{\mathbb{Q}} \mathscr{Q}\left(\mathbf{w}_{1}, \varsigma\right)+\overline{\mathbb{N}} \mathscr{Q}\left(\mathbf{w}_{1}, \varsigma\right)\right] \\
& +\frac{\psi^{\vartheta}(\varsigma)(1-\vartheta)+\vartheta}{\mathbb{A}(\vartheta) \psi^{\vartheta}(\varsigma)} \mathbb{J}\left[\mathbf{F}\left(\mathbf{w}_{1}, \varsigma\right)\right]
\end{aligned}
$$

By the virtue of the inverse Jafari transform of (30) and (31), respectively, this yields

$$
\begin{aligned}
\mathscr{Q}\left(\mathbf{w}_{1}, \varsigma\right)= & \mathscr{G}(\psi)+\mathbb{J}^{-1}\left\{\frac{1}{\psi^{9}(\varsigma)} \mathbb{J}\left[\mathbf{F}\left(\mathbf{w}_{1}, \varsigma\right)\right]\right\} \\
& -\mathbb{J}^{-1}\left\{\frac{1}{\psi^{9}(\varsigma)} \mathbb{J}\left[\overline{\mathbb{L}} \mathbb{Q}\left(\mathbf{w}_{1}, \varsigma\right)+\overline{\mathbb{N}} \mathscr{Q}\left(\mathbf{w}_{1}, \varsigma\right)\right]\right\} \\
\mathscr{Q}\left(\mathbf{w}_{1}, \varsigma\right)= & \mathscr{G}(\psi)+\mathbb{J}^{-1}\left\{\frac{\psi^{9}(\varsigma)(1-\vartheta)+\vartheta}{\mathbb{A}(\vartheta) \psi^{9}(\varsigma)} \mathbb{J}\left[\mathbf{F}\left(\mathbf{w}_{1}, \varsigma\right)\right]\right\} \\
& -\mathbb{J}^{-1}\left\{\frac{\psi^{9}(\varsigma)(1-\vartheta)+\vartheta}{\mathbb{A}(\vartheta) \psi^{9}(\varsigma)} \mathbb{J}\left[\overline{\mathbb{Q} Q}\left(\mathbf{w}_{1}, \varsigma\right)+\overline{\mathbb{N}} Q\left(\mathbf{w}_{1}, \varsigma\right)\right]\right\}
\end{aligned}
$$

Using the fact of an iterative process, we find

$$
Q\left(\mathbf{w}_{1}, \varsigma\right)=\sum_{q=0}^{\infty} Q_{q}\left(\mathbf{w}_{1}, \varsigma\right)
$$

Also, the operator $\overline{\mathbb{L}}$ is linear; therefore,

$$
\overline{\mathbb{L}}\left(\sum_{q=0}^{\infty} \widehat{Q}_{q}\left(\mathbf{w}_{1}, \varsigma\right)\right)=\sum_{q=0}^{\infty} \overline{\mathbb{L}}\left[\widehat{Q}_{q}\left(\mathbf{w}_{1}, \varsigma\right)\right],
$$

and the nonlinearity $\overline{\mathbb{N}}$ dealt by (see [27])

$$
\begin{aligned}
\overline{\mathbb{N}}\left(\sum_{q=0}^{\infty} \widehat{Q}_{q}\left(\mathbf{w}_{1}, \varsigma\right)\right)= & \overline{\mathbb{N}}\left(\mathcal{Q}_{0}\left(\mathbf{w}_{1}, \varsigma\right)\right)+\sum_{q=1}^{\infty}\left[\overline{\mathbb{N}}\left(\sum_{\kappa=0}^{q} \widehat{Q}_{q}\left(\mathbf{w}_{1}, \varsigma\right)\right)\right. \\
& \left.-\overline{\mathbb{N}}\left(\sum_{\kappa=0}^{q-1} \widehat{Q}_{q}\left(\mathbf{w}_{1}, \varsigma\right)\right)\right] \\
= & \overline{\mathbb{N}}\left(\mathcal{Q}_{0}\right)+\sum_{q=1}^{\infty} D_{q}
\end{aligned}
$$

where $D_{q}=\overline{\mathbb{N}}\left(\sum_{\kappa=0}^{q} Q_{q}\right)-\overline{\mathbb{N}}\left(\sum_{\kappa=0}^{q-1} Q_{q}\right)$.

Plugging (37), (39), and (36) into (32) and (33), respectively, we attain

$$
\begin{aligned}
\sum_{q=0}^{\infty} \mathbb{Q}_{q}\left(\mathbf{w}_{1}, \varsigma\right)= & \mathscr{S}(\psi)+\mathbb{J}^{-1}\left\{\frac{1}{\psi^{9}(\varsigma)} \mathbb{J}\left[\mathbf{F}\left(\mathbf{w}_{1}, \varsigma\right)\right]\right\}, \\
& -\mathbb{J}^{-1}\left\{\frac { 1 } { \psi ^ { 9 } ( \varsigma ) } \mathbb { \mathbb { L } } \left[\overline{\mathbb{L}}\left(\sum_{\kappa=0}^{q} \widehat{Q}_{q}\left(\mathbf{w}_{1}, \varsigma\right)\right)\right.\right. \\
& \left.\left.+\overline{\mathbb{N}}\left(\mathbb{Q}_{0}\right)+\sum_{\kappa=1}^{q} D_{q}\right]\right\}
\end{aligned}
$$

$$
\begin{aligned}
\sum_{q=0}^{\infty} \mathcal{Q}_{q}\left(\mathbf{w}_{1}, \varsigma\right)= & \mathscr{G}(\psi)+\mathbb{J}^{-1}\left\{\frac{\psi^{\vartheta}(\varsigma)(1-\vartheta)+\vartheta}{\mathbb{A}(\vartheta) \psi^{\vartheta}(\varsigma)} \mathbb{J}\left[\mathbf{F}\left(\mathbf{w}_{1}, \varsigma\right)\right]\right\}, \\
& -\mathbb{J}^{-1}\left\{\frac{\psi^{\vartheta}(\varsigma)(1-\vartheta)+\vartheta}{\mathbb{A}(\vartheta) \psi^{\vartheta}(\varsigma)} \mathbb{\mathbb { L }}\left[\sum_{q=0}^{q} \mathbb{Q}_{q}\left(\mathbf{w}_{1}, \varsigma\right)\right)\right. \\
& \left.\left.+\overline{\mathbb{N}}\left(\mathcal{Q}_{0}\right)+\sum_{q=1}^{q} D_{q}\right]\right\} .
\end{aligned}
$$
CFD:

Finally, we derive the following iterative process for 


$$
\begin{gathered}
\mathscr{Q}_{0}\left(\boldsymbol{w}_{1}, \varsigma\right)=\mathscr{G}(\psi)+\mathbb{J}^{-1}\left\{\frac{1}{\psi^{9}(\varsigma)} \mathscr{L}\left[\boldsymbol{F}\left(\boldsymbol{w}_{1}, \varsigma\right)\right]\right\} \\
\mathcal{Q}_{1}\left(\boldsymbol{w}_{1}, \varsigma\right)=-\mathbb{J}^{-1}\left\{\frac{1}{\psi^{9}(\varsigma)} \mathbb{\mathbb { C }}\left[\overline{\mathbb{L}}\left(\mathcal{Q}_{0}\left(\boldsymbol{w}_{1}, \varsigma\right)\right)+\overline{\mathbb{N}}\left(\mathcal{Q}_{0}\left(\boldsymbol{w}_{1}, \varsigma\right)\right)\right]\right\} \\
\vdots \\
\mathbb{Q}_{q+1}\left(\boldsymbol{w}_{1}, \varsigma\right)=-\mathbb{J}^{-1}\left\{\frac{1}{\psi^{9}(\varsigma)} \mathbb{J}\left[\overline{\mathbb{L}}\left(\mathcal{Q}_{q}\left(\boldsymbol{w}_{1}, \varsigma\right)\right)+D_{q}\right]\right\}
\end{gathered}
$$

Again, the iterative process for $\mathrm{ABC}$ fractional derivative operator is presented as follows:

$$
\begin{gathered}
\mathscr{Q}_{0}\left(\boldsymbol{w}_{1}, \varsigma\right)=\mathscr{G}(\psi)+\mathbb{J}^{-1}\left\{\frac{\psi^{\vartheta}(\varsigma)(1-\vartheta)+\vartheta}{\mathbb{A}(\vartheta) \psi^{9}(\varsigma)} \mathscr{L}\left[\boldsymbol{F}\left(\boldsymbol{w}_{1}, \varsigma\right)\right]\right\}, \\
\mathscr{Q}_{1}\left(\boldsymbol{w}_{1}, \varsigma\right)=-\mathbb{J}^{-1}\left\{\frac{\psi^{\vartheta}(\varsigma)(1-\vartheta)+\vartheta}{\mathbb{A}(\vartheta) \psi^{9}(\varsigma)} \mathbb{J}\left[\overline{\mathbb{L}}\left(\mathbb{Q}_{0}\left(\boldsymbol{w}_{1}, \varsigma\right)\right)+\overline{\mathbb{N}}\left(\mathscr{Q}_{0}\left(\boldsymbol{w}_{1}, \varsigma\right)\right)\right]\right\}, \\
\vdots \\
\widehat{Q}_{q+1}\left(\boldsymbol{w}_{1}, \varsigma\right)=-\mathbb{J}^{-1}\left\{\frac{\psi^{\vartheta}(\varsigma)(1-\vartheta)+\vartheta}{\mathbb{A}(\vartheta) \psi^{9}(\varsigma)} \mathbb{J}\left[\overline{\mathbb{L}}\left(\mathbb{Q}_{q}\left(\boldsymbol{w}_{1}, \varsigma\right)\right)+D_{q}\right]\right\} .
\end{gathered}
$$

Finally, (37), (39), and (40) produce the q-term solution in series representation, stated as

$$
\mathbb{Q}\left(\mathbf{w}_{1}, \varsigma\right) \neg \mathbb{Q}_{0}\left(\mathbf{w}_{1}, \varsigma\right)+\mathbb{Q}_{1}\left(\mathbf{w}_{1}, \varsigma\right)+\mathbb{Q}_{2}\left(\mathbf{w}_{1}, \varsigma\right)+\cdots+\mathbb{Q}_{q}\left(\mathbf{w}_{1}, \varsigma\right), q \in \mathbb{N} .
$$

\section{Convergence and Uniqueness Analyses of BSM via ABC Fractional Derivative Operator}

The subsequent subsections will highlight how sufficient requirements guarantee the emergence of a unique solution. Our anticipated existence of solutions in the case of JDM is followed by [53].

Theorem 12 (uniqueness theorem). Equation (24) has a unique solution whenever $0<\varepsilon<1$, where $\varepsilon=\left(\mathscr{K}_{1}+\mathscr{K}_{2}+\right.$ $\left.\mathscr{K}_{3}\right) / \mathbb{A}(\vartheta)\left[\vartheta\left(\varsigma^{(\vartheta)} / \Gamma(\vartheta+1)\right)+(1-\vartheta)\right]$.

Proof. Assuming all continuous functions on the Banach space are denoted by $\Omega=(\mathbb{C}[\mathscr{I}],\|\bullet\|)$. Also, suppose that $\mathscr{I}=[0, \mathscr{T}]$ have the norm $\|\bullet\|$. Now, we define a function $\mathscr{V}: \Omega \mapsto \Omega$ such that

$$
\begin{aligned}
\mathcal{Q}_{\ell+1}\left(\mathbf{w}_{1}, \varsigma\right)= & \mathscr{Q}\left(\mathbf{w}_{1}, \varsigma\right)+\mathbb{J}^{-1}\left[\frac { \psi ^ { \vartheta } ( \varsigma ) ( 1 - \vartheta ) + \vartheta } { \mathbb { A } ( \vartheta ) \psi ^ { 9 } ( \varsigma ) } \mathbb { I } \left[\mathbb{L}\left[\mathcal{Q}_{\ell}\left(\mathbf{w}_{1}, \varsigma\right)\right]\right.\right. \\
& \left.+\bar{P}\left[\mathcal{Q}_{\ell}\left(\mathbf{w}_{1}, \varsigma\right)\right]+\mathbb{N}\left[\mathcal{Q}_{\ell}\left(\mathbf{w}_{1}, \varsigma\right)\right]\right], \ell \geq 0,
\end{aligned}
$$

where $\mathbb{L}\left[Q\left(\mathbf{w}_{1}, \varsigma\right)\right] \equiv \partial^{3} Q\left(\mathbf{w}_{1}, \varsigma\right) / \partial \mathbf{w}_{1}^{2}$ and $\bar{P}\left[Q\left(\mathbf{w}_{1}, \varsigma\right)\right] \equiv \partial Q($ $\left.\mathbf{w}_{1}, \varsigma\right) / \partial \mathbf{w}_{1}$. Here, suppose that $\mathbb{L}\left[Q\left(\mathbf{w}_{1}, \varsigma\right)\right]$ and $\mathbb{N}\left[Q\left(\mathbf{w}_{1}, \varsigma\right)\right]$ are also Lipschitzian with $|\bar{P} Q-\bar{P} \widehat{Q}|<\mathscr{K}_{1}|Q-\widehat{Q}|$ and $\mid \mathbb{L} Q$ $-\mathbb{Q} \widehat{Q}\left|<\mathscr{K}_{2}\right| \mathscr{Q}-\widehat{Q} \mid$, where $\mathscr{K}_{1}$ and $\mathscr{K}_{2}$ are Lipschitz constant, respectively, and $\mathbb{Q}, \widehat{Q}$ are distinct functional values.

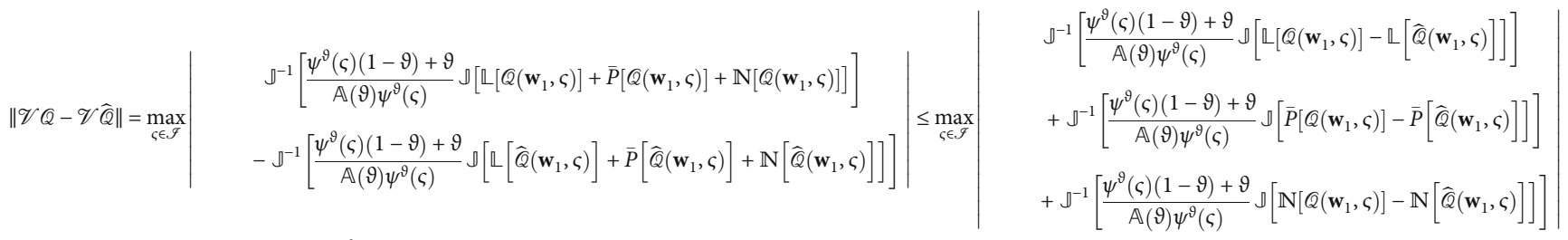

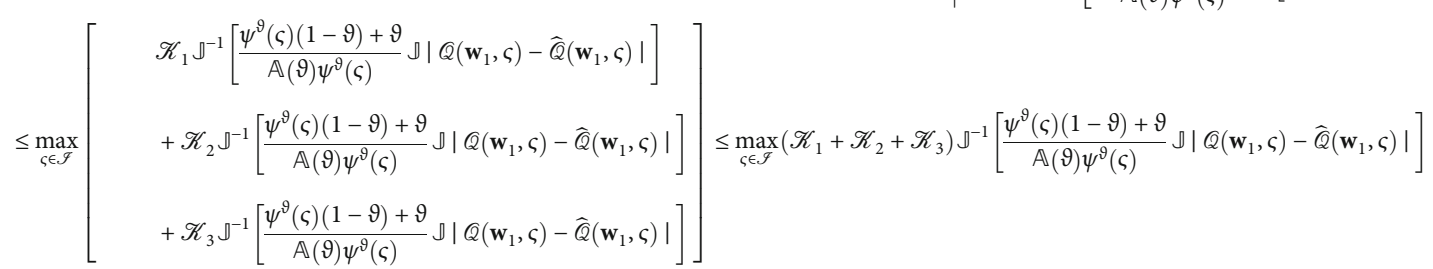

$$
\begin{aligned}
& \leq\left(\mathscr{K}_{1}+\mathscr{K}_{2}+\mathscr{K}_{3}\right) \mathbb{D}^{-1}\left[\frac{\psi^{9}(\varsigma)(1-\vartheta)+\vartheta}{\mathbb{A}(\vartheta) \psi^{9}(\varsigma)} \rrbracket\left\|\mathscr{Q}\left(\mathbf{w}_{1}, \varsigma\right)-\widehat{Q}\left(\mathbf{w}_{1}, \varsigma\right)\right\|\right]=\left(\mathscr{K}_{1}+\mathscr{K}_{2}+\mathscr{K}_{3}\right) \rrbracket^{-1}\left[\frac{\psi^{9}(\varsigma)(1-\vartheta)+\vartheta}{\mathbb{A}(\vartheta) \psi^{9}(\varsigma)} \frac{\phi(\mathfrak{g})}{\psi(\mathfrak{g})}\left\|Q\left(\mathbf{w}_{1}, \varsigma\right)-\widehat{Q}\left(\mathbf{w}_{1}, \varsigma\right)\right\|\right] \\
& =\frac{\left(\mathscr{K}_{1}+\mathscr{K}_{2}+\mathscr{K}_{3}\right)}{\mathbb{A}(\vartheta)}\left[\vartheta \frac{\varsigma^{(\vartheta)}}{\Gamma(\vartheta+1)}+(1-\vartheta)\right]\left\|Q\left(\mathbf{w}_{1}, \varsigma\right)-\widehat{Q}\left(\mathbf{w}_{1}, \varsigma\right)\right\| .
\end{aligned}
$$


Since $0<\varepsilon<1$, the mapping is contraction. Consequently, by the Banach contraction fixed point theorem, (17) has a unique. This gives the desired result.

Theorem 13 (convergence analysis). The general form solution of (17) will be convergent.

Proof. Assume that $\widehat{W}_{\ell}$ be the $n$th partial sum, i.e., $\widehat{W}_{\ell}=$ $\sum_{m=0}^{\ell} Q_{\ell}\left(\mathbf{w}_{1}, \varsigma\right)$. Here, we prove that a Cauchy sequence $\left\{\widehat{W}_{\ell}\right\}$ in Banach space $U$.

We acquire by considering a new form of Adomian polynomials.

$$
\begin{aligned}
& \bar{R}\left(\widehat{W}_{\ell}\right)=\tilde{\mathscr{H}}_{\ell}+\sum_{p=0}^{\ell-1} \tilde{\mathscr{H}}_{p}, \\
& \mathbb{N}\left(\widehat{W}_{\ell}\right)=\tilde{\mathscr{H}}_{\ell}+\sum_{c=0}^{\ell-1} \tilde{\mathscr{H}}_{c} .
\end{aligned}
$$

Now,

$$
\begin{aligned}
& \left\|\widehat{W}_{\ell}-\widehat{W}_{q}\right\|=\max _{\varsigma \in \mathscr{Y}}\left|\widehat{W}_{\ell}-\widehat{W}_{q}\right|=\max _{\varsigma \in \mathscr{Y}}\left|\sum_{m=q+1}^{\ell} \widehat{Q}\left(\mathbf{w}_{1}, \varsigma\right)\right|,(m=1,2,3, \cdots) \\
& \leq \max _{\varsigma \in \mathcal{G}} \mid J^{-1}\left[\frac{\psi^{9}(\varsigma)(1-\vartheta)+\vartheta}{\mathbb{A}(\vartheta) \psi^{\vartheta}(\varsigma)} \sqrt{ }\left[\sum_{m=q+1}^{\ell} \mathbb{L}\left[Q_{\ell-1}\left(\mathbf{w}_{1}, \varsigma\right)\right]\right]\right] \\
& +\rrbracket^{-1}\left[\frac{\psi^{9}(\varsigma)(1-\vartheta)+\vartheta}{A(\vartheta) \psi^{9}(\varsigma)} \rrbracket\left[\sum_{m=q+1}^{\ell} \bar{P}\left[Q_{\ell-1}\left(\mathbf{w}_{1}, \varsigma\right)\right]\right]\right] \\
& +\mathbb{J}^{-1}\left[\frac{\psi^{9}(\varsigma)(1-\vartheta)+\vartheta}{\mathbb{A}(\vartheta) \psi^{\vartheta}(\varsigma)} \rrbracket\left[\sum_{m=q+1}^{\ell} \tilde{\mathscr{H}}_{\ell-1}\left(\mathbf{w}_{1}, \varsigma\right)\right]\right] \mid \\
& =\max _{\varsigma \in \mathcal{Y}} \mid \rrbracket^{-1}\left[\frac{\psi^{\vartheta}(\varsigma)(1-\vartheta)+\vartheta}{\mathbb{A}(\vartheta) \psi^{\vartheta}(\varsigma)} \rrbracket\left[\sum_{m=q}^{\ell-1} \mathbb{L}\left[Q_{\ell}\left(\mathbf{w}_{1}, \varsigma\right)\right]\right]\right] \\
& +\mathbb{J}^{-1}\left[\frac{\psi^{\vartheta}(\varsigma)(1-\vartheta)+\vartheta}{\mathbb{A}(\vartheta) \psi^{9}(\varsigma)} \sqrt{ }\left[\sum_{m=q}^{\ell-1} \bar{P}\left[Q_{\ell}\left(\mathbf{w}_{1}, \varsigma\right)\right]\right]\right] \\
& +\mathbb{J}^{-1}\left[\frac{\psi^{9}(\varsigma)(1-\vartheta)+\vartheta}{\mathbb{A}(\vartheta) \psi^{9}(\varsigma)} \rrbracket\left[\sum_{m=q}^{\ell-1} \tilde{\mathscr{H}}_{\ell}\left(\mathbf{w}_{1}, \varsigma\right)\right]\right] \mid \\
& \leq \max _{\varsigma \in \mathcal{G}} \mid \mathbb{D}^{-1}\left[\frac{\psi^{\vartheta}(\varsigma)(1-\vartheta)+\vartheta}{\mathbb{A}(\vartheta) \psi^{9}(\varsigma)} \rrbracket\left[\sum_{m=q}^{\ell-1} \mathbb{L}\left(\widehat{W}_{\ell-1}\right)-\mathbb{L}\left(\widehat{W}_{q-1}\right)\right]\right] \\
& +\rrbracket^{-1}\left[\frac{\psi^{9}(\varsigma)(1-9)+\vartheta}{\mathbb{A}(\vartheta) \psi^{9}(\varsigma)} \rrbracket\left[\sum_{m=q}^{\ell-1} \bar{P}\left(\widehat{W}_{\ell-1}\right)-\bar{P}\left(\widehat{W}_{q-1}\right)\right]\right] \\
& +J^{-1}\left[\frac{\psi^{9}(\varsigma)(1-\vartheta)+\vartheta}{\mathbb{A}(\vartheta) \psi^{9}(\varsigma)} \rrbracket\left[\sum_{m=q}^{\ell-1} \mathbb{N}\left(\widehat{W}_{\ell-1}\right)-\mathbb{N}\left(\widehat{W}_{q-1}\right)\right]\right] \mid \\
& \leq \max _{\varsigma \in \mathcal{Y}} \mid \rrbracket^{-1}\left[\frac{\psi^{9}(\varsigma)(1-\vartheta)+\vartheta}{\mathbb{A}(\vartheta) \psi^{9}(\varsigma)} \rrbracket\left[\mathbb{L}\left(\widehat{W}_{\ell-1}\right)-\mathbb{L}\left(\widehat{W}_{q-1}\right)\right]\right] \\
& +\rrbracket^{-1}\left[\frac{\psi^{9}(\varsigma)(1-\vartheta)+\vartheta}{\mathbb{A}(\vartheta) \psi^{9}(\varsigma)} \rrbracket\left[\bar{P}\left(\widehat{W}_{\ell-1}\right)-\bar{P}\left(\widehat{W}_{q-1}\right)\right]\right] \\
& +\rrbracket^{-1}\left[\frac{\psi^{\vartheta}(\varsigma)(1-\vartheta)+\vartheta}{\mathbb{A}(\vartheta) \psi^{9}(\varsigma)} \rrbracket\left[\mathbb{N}\left(\widehat{W}_{\ell-1}\right)-\mathbb{N}\left(\widehat{W}_{q-1}\right)\right]\right] \\
& \leq \mathscr{K}_{1} \max _{\zeta \in \mathcal{I}} \rrbracket^{-1}\left|\left[\frac{\psi^{9}(\varsigma)(1-\vartheta)+\vartheta}{A(\vartheta) \psi^{9}(\varsigma)} \rrbracket\left[\left(\widehat{W}_{\ell-1}\right)-\left(\widehat{W}_{q-1}\right)\right]\right]\right|
\end{aligned}
$$

$$
\begin{aligned}
& +\mathscr{K}_{2} \max _{\varsigma \in \mathcal{I}}\left|\mathbb{J}^{-1}\left[\frac{\psi^{9}(\varsigma)(1-\vartheta)+\vartheta}{A(\vartheta) \psi^{9}(\varsigma)} \rrbracket\left[\left(\widehat{W}_{\ell-1}\right)-\left(\widehat{W}_{q-1}\right)\right]\right]\right| \\
& +\mathscr{K}_{3} \max _{\varsigma \in \mathcal{I}}\left|\rrbracket^{-1}\left[\frac{\psi^{9}(\varsigma)(1-\vartheta)+\vartheta}{\mathbb{A}(9) \psi^{9}(\varsigma)} \rrbracket\left[\left(\widehat{W}_{\ell-1}\right)-\left(\widehat{W}_{q-1}\right)\right]\right]\right| \\
& =\left(\mathscr{K}_{1}+\mathscr{K}_{2}+\mathscr{K}_{3}\right) \rrbracket^{-1}\left[\frac{\psi^{9}(\varsigma)(1-9)+\vartheta}{\mathbb{A}(\mathcal{9}) \psi^{9}(\varsigma)} \frac{\phi(\mathfrak{g})}{\psi^{9}(\mathfrak{g})}\left\|\widehat{W}_{\ell-1}-\widehat{W}_{q-1}\right\| .\right. \\
& =\frac{\left(\mathscr{K}_{1}+\mathscr{K}_{2}+\mathscr{K}_{3}\right)}{\mathrm{A}(\mathcal{\vartheta})}\left[\vartheta \frac{\varsigma^{\vartheta}}{\Gamma(\mathcal{\vartheta}+1)}+(1-\vartheta)\right]\left\|\widehat{W}_{\ell-1}-\widehat{W}_{q-1}\right\| .
\end{aligned}
$$

Consider $n=q+1$, then,

$$
\begin{aligned}
\left\|\widehat{W}_{q+1}-\widehat{W}_{q}\right\| & \leq \varepsilon\left\|\widehat{W}_{q}-\widehat{W}_{q-1}\right\| \leq \varepsilon^{2}\left\|\widehat{W}_{q-1}-\widehat{W}_{q-2}\right\| \\
& \leq \cdots \leq \varepsilon^{q}\left\|\widehat{W}_{1}-\widehat{W}_{0}\right\|,
\end{aligned}
$$

where $\varepsilon=\left(\mathscr{K}_{1}+\mathscr{K}_{2}+\mathscr{K}_{3}\right) / \mathbb{A}(\vartheta)\left[\vartheta\left(\varsigma^{\vartheta} / \Gamma(\vartheta+1)\right)+(1-\vartheta)\right]$. Now, from triangular inequality we have

$$
\begin{aligned}
\left\|\widehat{W}_{\ell}-\widehat{W}_{q}\right\| & \leq\left\|\widehat{W}_{q+1}-\widehat{W}_{q}\right\|+\left\|\widehat{W}_{q+2}-\widehat{W}_{q+1}\right\|+\cdots+\left\|\widehat{W}_{\ell}-\widehat{W}_{\ell-1}\right\| \\
& \leq\left[\varepsilon^{q}+\varepsilon^{q+1}+\cdots+\varepsilon^{\ell-1}\right]\left\|\widehat{W}_{1}-\widehat{W}_{0}\right\| \\
& \leq \varepsilon^{q}\left(\frac{1-\varepsilon^{\ell-q}}{\varepsilon}\right)\left\|Q_{1}\right\|,
\end{aligned}
$$

since $0<\varepsilon<1$, we have $\left(1-\varepsilon^{\ell-q}\right)<1$, then,

$$
\left\|\widehat{W}_{\ell}-\widehat{W}_{q}\right\| \leq \frac{\varepsilon^{q}}{1-\varepsilon} \max _{\varsigma \in \mathscr{I}}\left\|Q_{1}\right\| .
$$

Therefore, $\left|Q_{1}\right|<\infty$ (since $Q\left(\mathbf{w}_{1}, \varsigma\right)$ is bounded). Furthermore, as $q \mapsto \infty$, then $\left\|\widehat{W}_{\ell}-\widehat{W}_{q}\right\| \mapsto 0$. Thus, $\left\{\widehat{W}_{1}\right\}$ is a Cauchy sequence in $K$. Consequently, the series $\sum_{n=0}^{\infty} \mathbb{Q}_{\ell}$ is convergent and this yields the immediate consequence.

Theorem 14 (see [53]) (error estimate). The absolute error of the series solution (17) to (24) is calculated as

$$
\max _{\varsigma \in \mathscr{Y}}\left|Q_{(}\left(\mathbf{w}_{1}, \varsigma\right)-\sum_{\ell=1}^{q} Q_{\ell}\left(\mathbf{w}_{1}, \varsigma\right)\right| \leq \frac{\varepsilon^{q}}{1-\varepsilon} \max _{\varsigma \in \mathscr{I}}\left\|Q_{1}\right\|
$$

\section{Physical Interpretation of Time-Fractional Black-Scholes Models}

In this section, we compute the approximate analytical solution of $\mathrm{BSM}$ via the $\mathrm{CFD}$ and $\mathrm{ABC}$ fractional derivative operators by using the Jafari decomposition method.

\subsection{Jafari Decomposition Method}

Example 1 (see [16]). Assume the time-fractional onedimensional BSM (4) subject to IC (5). 
Case 1. Firstly, we solve the (4) by using Caputo fractional derivative operator incorporating the Jafari decomposition method.

Applying Jafari transform to both sides of (4), we have

$$
\mathbb{J}\left[\mathbf{D}_{\zeta}^{9} \mathscr{Q}\left(\mathbf{w}_{1}, \varsigma\right)\right]=\mathbb{J}\left[\frac{\partial^{2} \mathscr{Q}\left(\mathbf{w}_{1}, \varsigma\right)}{\partial \mathbf{w}_{1}^{2}}+(\eta-1) \frac{\partial \mathscr{Q}\left(\mathbf{w}_{1}, \varsigma\right)}{\partial \mathbf{w}_{1}}-\eta \mathscr{Q}\left(\mathbf{w}_{1}, \varsigma\right)\right] .
$$
have

Using the differentiation rule of Jafari transform, we

$$
\begin{aligned}
& \psi^{9}(\varsigma) \mathbb{J}\left[\mathcal{Q}\left(\mathbf{w}_{1}, \varsigma\right)\right]=\phi(\varsigma) \sum_{q=0}^{q-1} \psi^{9-1-q}(\varsigma) Q^{(q)}(0) \\
& +\sqrt{ }\left[\frac{\partial^{2} \mathscr{Q}\left(\mathbf{w}_{1}, \varsigma\right)}{\partial \mathbf{w}_{1}^{2}}+(\eta-1) \frac{\partial \mathscr{Q}\left(\mathbf{w}_{1}, \varsigma\right)}{\partial \mathbf{w}_{1}}-\eta \mathscr{Q}\left(\mathbf{w}_{1}, \varsigma\right)\right] .
\end{aligned}
$$

In view of (5), we get

$$
\begin{aligned}
\mathbb{D}\left[\mathscr{Q}\left(\mathbf{w}_{1}, \varsigma\right)\right] & =\frac{\phi(\varsigma)}{\psi^{9}(\varsigma)} \max \left(\exp \left(\mathbf{w}_{1}\right)-1,0\right) \\
& +\frac{1}{\psi^{9}(\varsigma)} \rrbracket\left[\frac{\partial^{2} \mathscr{Q}\left(\mathbf{w}_{1}, \varsigma\right)}{\partial \mathbf{w}_{1}^{2}}+(\eta-1) \frac{\partial Q\left(\mathbf{w}_{1}, \varsigma\right)}{\partial \mathbf{w}_{1}}-\eta \mathbb{Q}\left(\mathbf{w}_{1}, \varsigma\right)\right] .
\end{aligned}
$$
yields

Employing the inverse Jafari transform on both sides

$$
\begin{aligned}
\mathscr{Q}\left(\mathbf{w}_{1}, \varsigma\right)= & \mathbb{J}^{-1}\left[\frac{\phi(\varsigma)}{\psi^{9}(\varsigma)} \max \left(\exp \left(\mathbf{w}_{1}\right)-1,0\right)\right] \\
& +\mathbb{J}^{-1}\left[\frac{1}{\psi^{9}(\varsigma)} \sqrt{ }\left[\frac{\partial^{2} \mathscr{Q}\left(\mathbf{w}_{1}, \varsigma\right)}{\partial \mathbf{w}_{1}^{2}}+(\eta-1) \frac{\partial \mathscr{Q}\left(\mathbf{w}_{1}, \varsigma\right)}{\partial \mathbf{w}_{1}}-\eta \mathbb{Q}\left(\mathbf{w}_{1}, \varsigma\right)\right]\right] .
\end{aligned}
$$

With the help of Jafari decomposition method, we find

$$
\begin{aligned}
\mathbb{Q}_{0}\left(\mathbf{w}_{1}, \varsigma\right) & =\mathbb{J}^{-1}\left[\frac{\phi(\varsigma)}{\psi^{9}(\varsigma)} \max \left(\exp \left(\mathbf{w}_{1}\right)-1,0\right)\right] \\
& =\max \left(\exp \left(\mathbf{w}_{1}\right)-1,0\right) .
\end{aligned}
$$

Here, we surmise that the unknown function $Q\left(\mathbf{w}_{1}, \varsigma\right)$ can be written by an infinite series of the form

$$
\mathcal{Q}\left(\mathbf{w}_{1}, \varsigma\right)=\sum_{q=0}^{\infty} \widehat{Q}_{q}\left(\mathbf{w}_{1}, \varsigma\right)
$$

$$
\begin{aligned}
& \sum_{q=0}^{\infty} \widehat{Q}_{q+1}\left(\mathbf{w}_{1}, \varsigma\right)=\mathbb{J}^{-1}\left[\frac { 1 } { \psi ^ { 9 } ( \varsigma ) } \mathbb { \mathbb { S } } \left[\sum_{q=0}^{\infty}\left(\mathcal{Q}\left(\mathbf{w}_{1}, \varsigma\right)\right)_{\mathbf{w}_{1} \mathbf{w}_{1}}\right.\right. \\
& \left.\left.+(\eta-1) \sum_{q=0}^{\infty}\left(Q\left(\mathbf{w}_{1}, \varsigma\right)\right)_{\mathbf{w}_{1}}-\eta \sum_{q=0}^{\infty}\left(\mathcal{Q}\left(\mathbf{w}_{1}, \varsigma\right)\right)\right]\right] \text {, } \\
& q=0,1,2, \cdots, \\
& \mathbb{Q}_{1}\left(\mathbf{w}_{1}, \varsigma\right)=\mathbb{J}^{-1}\left[\frac{1}{\psi^{9}(\varsigma)} \mathbb{J}\left[\left(\mathbb{Q}_{0}\left(\mathbf{w}_{1}, \varsigma\right)\right)_{\mathbf{w}_{1} \mathbf{w}_{1}}+(\eta-1)\left(\mathbb{Q}_{0}\left(\mathbf{w}_{1}, \varsigma\right)\right)_{\mathbf{w}_{1}}+\eta Q_{0}\right]\right] \\
& =\left[\eta \max \left(\exp \left(\mathbf{w}_{1}\right), 0\right)-\eta \max \left(\exp \left(\mathbf{w}_{1}-1\right), 0\right)\right] \mathbb{D}^{-1}\left[\frac{\phi(\mathfrak{g})}{\psi^{9+1}(\mathfrak{g})}\right] \\
& =\left[\eta \max \left(\exp \left(\mathbf{w}_{1}\right), 0\right)-\eta \max \left(\exp \left(\mathbf{w}_{1}-1\right), 0\right)\right] \frac{\varsigma^{\vartheta}}{\Gamma(\vartheta+1)}, \\
& \begin{aligned}
\mathbb{Q}_{2}\left(\mathbf{w}_{1}, \varsigma\right) & =\mathbb{J}^{-1}\left[\frac{1}{\psi^{9}(\varsigma)} \sqrt{ }\left[\left(\mathbb{Q}_{1}\left(\mathbf{w}_{1}, \varsigma\right)\right)_{\mathbf{w}_{1} \mathbf{w}_{1}}+(\eta-1)\left(\mathbb{Q}_{1}\left(\mathbf{w}_{1}, \varsigma\right)\right)_{\mathbf{w}_{1}}+\eta Q_{1}\right]\right] \\
& =\left[-\eta^{2} \max \left(\exp \left(\mathbf{w}_{1}\right), 0\right)+\eta^{2} \max \left(\exp \left(\mathbf{w}_{1}-1\right), 0\right)\right] \frac{\varsigma^{29}}{\Gamma(2 \vartheta+1)}, \\
\mathbb{Q}_{3}\left(\mathbf{w}_{1}, \varsigma\right) & =\mathbb{J}^{-1}\left[\frac{1}{\psi^{9}(\varsigma)} \sqrt{ }\left[\left(\mathcal{Q}_{2}\left(\mathbf{w}_{1}, \varsigma\right)\right)_{\mathbf{w}_{1} \mathbf{w}_{1}}+(\eta-1)\left(\mathscr{Q}_{2}\left(\mathbf{w}_{1}, \varsigma\right)\right)_{\mathbf{w}_{1}}+\eta \mathscr{Q}_{2}\right]\right] \\
& =\left[-\eta^{3} \max \left(\exp \left(\mathbf{w}_{1}\right), 0\right)+\eta^{3} \max \left(\exp \left(\mathbf{w}_{1}-1\right), 0\right)\right] \frac{\varsigma^{39}}{\Gamma(3 \vartheta+1)} .
\end{aligned}
\end{aligned}
$$$$
\vdots
$$

The approximate solution for Example 1 is expressed as

$$
\begin{aligned}
Q\left(\mathbf{w}_{1}, \varsigma\right)= & \mathcal{Q}_{0}\left(\mathbf{w}_{1}, \varsigma\right)+Q_{1}\left(\mathbf{w}_{1}, \varsigma\right)+Q_{2}\left(\mathbf{w}_{1}, \varsigma\right)+Q_{3}\left(\mathbf{w}_{1}, \varsigma\right)+\cdots \\
= & \max \left(\exp \left(\mathbf{w}_{1}-1\right), 0\right)\left[1-\frac{\eta \varsigma^{\vartheta}}{\Gamma(\vartheta+1)}+\frac{\eta^{2} \varsigma^{2 \vartheta}}{\Gamma(2 \vartheta+1)}\right. \\
& \left.-\frac{\eta^{3} \varsigma^{3 \vartheta}}{\Gamma(3 \vartheta+1)}+\cdots\right]+\max \left(\exp \left(\mathbf{w}_{1}\right), 0\right) \\
& \cdot\left[1-1+\frac{\eta \varsigma^{\vartheta}}{\Gamma(\vartheta+1)}-\frac{\eta^{2} \varsigma^{2 \vartheta}}{\Gamma(2 \vartheta+1)}+\frac{\eta^{3} \varsigma^{3 \vartheta}}{\Gamma(3 \vartheta+1)}+\cdots\right] \\
= & \max \left(\exp \left(\mathbf{w}_{1}-1\right), 0\right) E_{\vartheta}\left(-\eta(\varsigma)^{\vartheta}\right) \\
& +\max \left(\exp \left(\mathbf{w}_{1}\right), 0\right) E_{\vartheta}\left(1-\eta(\varsigma)^{\vartheta}\right) .
\end{aligned}
$$

Case 2. Now, we solve(4) by using the ABC fractional derivative operator incorporating the Jafari decomposition method.

Considering (50) and using the differentiation rule of Jafari transform, we have

$$
\begin{gathered}
\frac{\psi^{\vartheta}(\varsigma) \mathbb{A}(\vartheta)}{\vartheta+(1-\vartheta) \psi^{9}(\varsigma)} \mathbb{J}\left[\mathscr{Q}\left(\mathbf{w}_{1}, \varsigma\right)\right]=\phi(\varsigma) \sum_{q=0}^{q-1} \psi^{9-1-q}(\varsigma) Q^{(q)}(0) \\
+\mathbb{J}\left[\frac{\partial^{2} \mathscr{Q}\left(\mathbf{w}_{1}, \varsigma\right)}{\partial \mathbf{w}_{1}^{2}}+(\eta-1) \frac{\partial \mathscr{Q}\left(\mathbf{w}_{1}, \varsigma\right)}{\partial \mathbf{w}_{1}}-\eta \mathbb{Q}\left(\mathbf{w}_{1}, \varsigma\right)\right] .
\end{gathered}
$$


In view of (5), we get

$$
\begin{aligned}
\mathbb{I}\left[\mathscr{Q}\left(\mathbf{w}_{1}, \varsigma\right)\right]= & \frac{\phi(\varsigma)}{\psi^{9}(\varsigma)} \max \left(\exp \left(\mathbf{w}_{1}\right)-1,0\right)+\frac{\vartheta+(1-\vartheta) \psi^{9}(\varsigma)}{\psi^{9}(\varsigma) \mathbb{A}(\vartheta)} \\
& \cdot \sqrt{\int}\left[\frac{\partial^{2} \mathscr{Q}\left(\mathbf{w}_{1}, \varsigma\right)}{\partial \mathbf{w}_{1}^{2}}+(\eta-1) \frac{\partial \mathscr{Q}\left(\mathbf{w}_{1}, \varsigma\right)}{\partial \mathbf{w}_{1}}-\eta \mathscr{Q}\left(\mathbf{w}_{1}, \varsigma\right)\right]
\end{aligned}
$$

Employing the inverse Jafari transform on both sides of the above equation yields

$$
\begin{aligned}
Q\left(\mathbf{w}_{1}, \varsigma\right)= & \mathbb{J}^{-1}\left[\frac{\phi(\varsigma)}{\psi^{9}(\varsigma)} \max \left(\exp \left(\mathbf{w}_{1}\right)-1,0\right)\right] \\
& +\mathbb{J}^{-1}\left[\frac { \vartheta + ( 1 - \vartheta ) \psi ^ { 9 } ( \varsigma ) } { \psi ^ { 9 } ( \varsigma ) \mathbb { A } ( \vartheta ) } \sqrt { \vartheta } \left[\frac{\partial^{2} \mathscr{Q}\left(\mathbf{w}_{1}, \varsigma\right)}{\partial \mathbf{w}_{1}^{2}}\right.\right. \\
& \left.\left.+(\eta-1) \frac{\partial \mathscr{Q}\left(\mathbf{w}_{1}, \varsigma\right)}{\partial \mathbf{w}_{1}}-\eta \mathscr{Q}\left(\mathbf{w}_{1}, \varsigma\right)\right]\right] .
\end{aligned}
$$

By the Jafari decomposition method, we find:

$$
\begin{aligned}
Q_{0}\left(\mathbf{w}_{1}, \varsigma\right) & =\mathbb{J}^{-1}\left[\frac{\phi(\varsigma)}{\psi^{9}(\varsigma)} \max \left(\exp \left(\mathbf{w}_{1}\right)-1,0\right)\right] \\
& =\max \left(\exp \left(\mathbf{w}_{1}\right)-1,0\right) .
\end{aligned}
$$

Here, we surmise that the unknown function $Q\left(\mathbf{w}_{1}, \varsigma\right)$ can be written by an infinite series of the form:

$$
\begin{aligned}
& Q\left(\mathbf{w}_{1}, \varsigma\right)=\sum_{q=0}^{\infty} \widehat{Q}_{\mathrm{q}}\left(\mathbf{w}_{1}, \varsigma\right) \\
& \sum_{q=0}^{\infty} \mathbb{Q}_{q+1}\left(\mathbf{w}_{1}, \varsigma\right)=\mathbb{J}^{-1}\left[\frac { \vartheta + ( 1 - \vartheta ) \psi ^ { \vartheta } ( \varsigma ) } { \psi ^ { \vartheta } ( \varsigma ) \mathbb { A } ( \vartheta ) } \sqrt { \vartheta } \left[\sum_{q=0}^{\infty}\left(\mathscr{Q}\left(\mathbf{w}_{1}, \varsigma\right)\right)_{\mathbf{w}_{1} \mathbf{w}_{1}}\right.\right. \\
& \left.\left.+(\eta-1) \sum_{q=0}^{\infty}\left(\mathbb{Q}\left(\mathbf{w}_{1}, \varsigma\right)\right)_{\mathbf{w}_{1}}-\eta \sum_{q=0}^{\infty}\left(\mathbb{Q}\left(\mathbf{w}_{1}, \varsigma\right)\right)\right]\right] \text {, } \\
& q=0,1,2, \cdots, \\
& \mathbb{Q}_{1}\left(\mathbf{w}_{1}, \varsigma\right)=\mathbb{J}^{-1}\left[\frac{\vartheta+(1-\vartheta) \psi^{9}(\varsigma)}{\psi^{9}(\varsigma) \mathbb{A}(\vartheta)} \mathbb{J}\left[\left(\mathscr{Q}_{0}\left(\mathbf{w}_{1}, \varsigma\right)\right)_{\mathbf{w}_{1} \mathbf{w}_{1}}+(\eta-1)\left(\mathbb{Q}_{0}\left(\mathbf{w}_{1}, \varsigma\right)\right)_{\mathbf{w}_{1}}+\eta Q_{0}\right]\right] \\
& =\frac{\left[\eta \max \left(\exp \left(\mathbf{w}_{1}\right), 0\right)-\eta \max \left(\exp \left(\mathbf{w}_{1}-1\right), 0\right)\right]}{\mathbb{A}(\vartheta)}\left[\vartheta \frac{\varsigma^{\vartheta}}{\Gamma(\vartheta+1)}+(1-\vartheta)\right], \\
& \mathbb{Q}_{2}\left(\mathbf{w}_{1}, \varsigma\right)=\rrbracket^{-1}\left[\frac{\vartheta+(1-\vartheta) \psi^{9}(\varsigma)}{\psi^{9}(\varsigma) \mathbb{A}(\vartheta)} \rrbracket\left[\left(\mathbb{Q}_{1}\left(\mathbf{w}_{1}, \varsigma\right)\right)_{\mathbf{w}_{1} \mathbf{w}_{1}}+(\eta-1)\left(\mathscr{Q}_{1}\left(\mathbf{w}_{1}, \varsigma\right)\right)_{\mathbf{w}_{1}}+\eta \mathscr{Q}_{1}\right]\right] \\
& =-\frac{\left[\eta^{2} \max \left(\exp \left(\mathbf{w}_{1}\right), 0\right)+\eta^{2} \max \left(\exp \left(\mathbf{w}_{1}-1\right), 0\right)\right]}{\mathbb{A}^{2}(9)} \\
& \cdot\left[\vartheta^{2} \frac{\varsigma^{2 \vartheta}}{\Gamma(2 \vartheta+1)}+2 \vartheta(1-\vartheta) \frac{\varsigma^{\vartheta}}{\Gamma(\vartheta+1)}+(1-\vartheta)^{2}\right] \text {, } \\
& \mathbb{Q}_{3}\left(\mathbf{w}_{1}, \varsigma\right)=\rrbracket^{-1}\left[\frac{\vartheta+(1-\vartheta) \psi^{9}(\varsigma)}{\psi^{9}(\varsigma) \mathbb{A}(\vartheta)} \rrbracket\left[\left(\mathbb{Q}_{2}\left(\mathbf{w}_{1}, \varsigma\right)\right)_{\mathbf{w}_{1} \mathbf{w}_{1}}+(\eta-1)\left(Q_{2}\left(\mathbf{w}_{1}, \varsigma\right)\right)_{\mathbf{w}_{1}}+\eta \mathbb{Q}_{2}\right]\right] \\
& =-\frac{\left[\eta^{3} \max \left(\exp \left(\mathbf{w}_{1}\right), 0\right)+\eta^{3} \max \left(\exp \left(\mathbf{w}_{1}-1\right), 0\right)\right]}{\mathbb{A}^{3}(9)} \\
& \times\left[\vartheta^{3} \frac{\varsigma^{39}}{\Gamma(39+1)}+3 \vartheta^{2}(1-\vartheta) \frac{\varsigma^{29}}{\Gamma(29+1)}+3 \vartheta(1-\vartheta)^{2} \frac{\varsigma^{9}}{\Gamma(9+1)}+(1-9)^{3}\right] \\
& \vdots
\end{aligned}
$$

The approximate solution for Example 1 is expressed as:

$$
\begin{aligned}
\mathscr{Q}\left(\mathbf{w}_{1}, \varsigma\right)= & \mathbb{Q}_{0}\left(\mathbf{w}_{1}, \varsigma\right)+\mathbb{Q}_{1}\left(\mathbf{w}_{1}, \varsigma\right)+\mathbb{Q}_{2}\left(\mathbf{w}_{1}, \varsigma\right)+\mathcal{Q}_{3}\left(\mathbf{w}_{1}, \varsigma\right)+\cdots \\
= & \max \left(\exp \left(\mathbf{w}_{1}-1\right), 0\right)\left[1-\frac{\eta}{\mathbb{A}(\vartheta)}\left[\vartheta \frac{\varsigma^{\vartheta}}{\Gamma(\vartheta+1)}+\vartheta\right]\right. \\
& \left.-\frac{\eta^{2}}{\mathbb{A}^{2}(\vartheta)}\left[\vartheta^{2} \frac{\varsigma^{2 \vartheta}}{\Gamma(2 \vartheta+1)}+2 \vartheta(1-\vartheta) \frac{\varsigma^{\vartheta}}{\Gamma(\vartheta+1)}+(1-\vartheta)^{2}\right]-\cdots\right] \\
& +\max \left(\exp \left(\mathbf{w}_{1}\right), 0\right)\left[-\frac{\eta}{\mathbb{A}(\vartheta)}\left[\vartheta \frac{\varsigma^{\vartheta}}{\Gamma(\vartheta+1)}+\vartheta\right]\right. \\
& \left.-\frac{\eta^{2}}{\mathbb{A}^{2}(\vartheta)}\left[\vartheta^{2} \frac{\varsigma^{2 \vartheta}}{\Gamma(2 \vartheta+1)}+2 \vartheta(1-\vartheta) \frac{\varsigma^{\vartheta}}{\Gamma(\vartheta+1)}+(1-\vartheta)^{2}\right]-\cdots\right] .
\end{aligned}
$$

The integer-order solution of Example 1 can be obtained with the aid of Taylor series expansion and setting $\vartheta=1$ as follows:

$$
\begin{aligned}
\mathcal{Q}\left(\mathbf{w}_{1}, \varsigma\right)= & \max \left(\exp \left(\mathbf{w}_{1}-1\right), 0\right) \exp (-\eta \varsigma) \\
& +\max \left(\exp \left(\mathbf{w}_{1}\right), 0\right)[1-\exp (-\eta \varsigma)] .
\end{aligned}
$$

Example 2 (see [20]). Assume the time-fractional onedimensional BSM (6) subject to IC (7).

Case 1. Firstly, we solve the (6) in the sense of Caputo fractional derivative operator incorporating the Jafari decomposition method.

Applying Jafari transform to both sides of (6), we have

$$
\begin{aligned}
\mathbb{J}\left[\mathbf{D}_{\varsigma}^{9} Q\left(\mathbf{w}_{1}, \varsigma\right)\right]= & \mathbb{J}\left[-0.08\left(2+\sin \mathbf{w}_{1}\right)^{2} \mathbf{w}_{1}^{2} \frac{\partial^{2} \mathscr{Q}\left(\mathbf{w}_{1}, \varsigma\right)}{\partial \mathbf{w}_{1}^{2}}\right. \\
& \left.-0.06 \mathbf{w}_{1} \frac{\partial \mathscr{Q}\left(\mathbf{w}_{1}, \varsigma\right)}{\partial \mathbf{w}_{1}}+0.06 \mathscr{Q}\left(\mathbf{w}_{1}, \varsigma\right)\right]
\end{aligned}
$$

Using the differentiation rule of Jafari transform, we have

$$
\begin{aligned}
& \psi^{9}(\varsigma) \mathbb{J}\left[\mathcal{Q}\left(\mathbf{w}_{1}, \varsigma\right)\right]=\phi(\varsigma) \sum_{q=0}^{q-1} \psi^{9-1-q}(\varsigma) Q^{(q)}(0) \\
& +\sqrt{ }\left[-0.08\left(2+\sin \mathbf{w}_{1}\right)^{2} \mathbf{w}_{1}^{2} \frac{\partial^{2} \mathscr{Q}\left(\mathbf{w}_{1}, \varsigma\right)}{\partial \mathbf{w}_{1}^{2}}\right. \\
& \left.-0.06 \mathbf{w}_{1} \frac{\partial Q\left(\mathbf{w}_{1}, \varsigma\right)}{\partial \mathbf{w}_{1}}+0.06 Q\left(\mathbf{w}_{1}, \varsigma\right)\right] \text {. }
\end{aligned}
$$

In view of (7), we get

$$
\begin{aligned}
& \mathbb{J}\left[Q\left(\mathbf{w}_{1}, \varsigma\right)\right]=\frac{\phi(\varsigma)}{\psi^{9}(\varsigma)} \max \left(\mathbf{w}_{1}-25 \exp (-0.06), 0\right)+\frac{1}{\psi^{9}(\varsigma)} \\
& \cdot \sqrt{\int}\left[-0.08\left(2+\sin \mathbf{w}_{1}\right)^{2} \mathbf{w}_{1}^{2} \frac{\partial^{2} \mathscr{Q}\left(\mathbf{w}_{1}, \varsigma\right)}{\partial \mathbf{w}_{1}^{2}}-0.06 \mathbf{w}_{1} \frac{\partial Q\left(\mathbf{w}_{1}, \varsigma\right)}{\partial \mathbf{w}_{1}}+0.06 \mathbb{Q}\left(\mathbf{w}_{1}, \varsigma\right)\right] .
\end{aligned}
$$



yields

Employing the inverse Jafari transform on both sides

$$
\begin{aligned}
\mathscr{Q}\left(\mathbf{w}_{1}, \varsigma\right)= & \mathbb{J}^{-1}\left[\frac{\phi(\varsigma)}{\psi^{9}(\varsigma)} \max \left(\mathbf{w}_{1}-25 \exp (-0.06), 0\right)\right] \\
& +\mathbb{J}^{-1}\left[\frac { 1 } { \psi ^ { 9 } ( \varsigma ) } \mathbb { J } \left[-0.08\left(2+\sin \mathbf{w}_{1}\right)^{2} \mathbf{w}_{1}^{2} \frac{\partial^{2} Q\left(\mathbf{w}_{1}, \varsigma\right)}{\partial \mathbf{w}_{1}^{2}}\right.\right. \\
& \left.\left.-0.06 \mathbf{w}_{1} \frac{\partial \mathscr{Q}\left(\mathbf{w}_{1}, \varsigma\right)}{\partial \mathbf{w}_{1}}+0.06 \mathbb{Q}\left(\mathbf{w}_{1}, \varsigma\right)\right]\right]
\end{aligned}
$$

By the Jafari decomposition method, we find

$$
\begin{aligned}
Q_{0}\left(\mathbf{w}_{1}, \varsigma\right) & =\mathbb{J}^{-1}\left[\frac{\phi(\varsigma)}{\psi^{9}(\varsigma)} \max \left(\mathbf{w}_{1}-25 \exp (-0.06), 0\right)\right] \\
& =\max \left(\mathbf{w}_{1}-25 \exp (-0.06), 0\right) .
\end{aligned}
$$

Here, we surmise that the unknown function $Q\left(\mathbf{w}_{1}, \varsigma\right)$ can be written by an infinite series of the form:

$$
\begin{aligned}
& Q\left(\mathbf{w}_{1}, \varsigma\right)=\sum_{q=0}^{\infty} \widehat{Q}_{q}\left(\mathbf{w}_{1}, \varsigma\right) \\
& \sum_{q=0}^{\infty} \mathbb{Q}_{q+1}\left(\mathbf{w}_{1}, \varsigma\right)=\mathbb{J}^{-1}\left[\frac { 1 } { \psi ^ { 9 } ( \varsigma ) } \sqrt [ J ] { } \left[-0.08\left(2+\sin \mathbf{w}_{1}\right)^{2} \mathbf{w}_{1}^{2} \sum_{q=0}^{\infty}\left(\mathscr{Q}\left(\mathbf{w}_{1}, \varsigma\right)\right)_{\mathbf{w}_{1} \mathbf{w}_{1}}\right.\right. \\
& \left.\left.-0.06 \mathbf{w}_{1} \sum_{q=0}^{\infty}\left(\mathscr{Q}\left(\mathbf{w}_{1}, \varsigma\right)\right)_{\mathbf{w}_{1}}+0.06 \sum_{q=0}^{\infty}\left(\mathscr{Q}\left(\mathbf{w}_{1}, \varsigma\right)\right)\right]\right] \text {, } \\
& \forall \mathrm{q}=0,1,2, \cdots, \\
& \mathbb{Q}_{1}\left(\mathbf{w}_{1}, \varsigma\right)=\mathbb{J}^{-1}\left[\frac { 1 } { \psi ^ { 9 } ( \varsigma ) } \mathbb { J } \left[-0.08\left(2+\sin \mathbf{w}_{1}\right)^{2} \mathbf{w}_{1}^{2}\left(\mathcal{Q}_{0}\left(\mathbf{w}_{1}, \varsigma\right)\right)_{\mathbf{w}_{1} \mathbf{w}_{1}}\right.\right. \\
& \left.\left.-0.06 \mathbf{w}_{1}\left(Q_{0}\left(\mathbf{w}_{1}, \varsigma\right)\right)_{\mathbf{w}_{1}}-0.06 Q_{0}\right]\right] \\
& =\left[-0.06 \mathbf{w}_{1}+0.06 \max \left(\mathbf{w}_{1}-25 \exp (-0.06), 0\right)\right] \mathbb{J}^{-1}\left[\frac{\phi(\mathfrak{I})}{\psi^{9+1}(\mathfrak{g})}\right] \\
& =\left[-0.06 \mathbf{w}_{1}+0.06 \max \left(\mathbf{w}_{1}-25 \exp (-0.06), 0\right)\right] \frac{\varsigma^{\vartheta}}{\Gamma(\vartheta+1)}, \\
& \mathbb{Q}_{2}\left(\mathbf{w}_{1}, \varsigma\right)=\mathbb{J}^{-1}\left[\frac { 1 } { \psi ^ { 9 } ( \varsigma ) } \mathbb { J } \left[-0.08\left(2+\sin \mathbf{w}_{1}\right)^{2} \mathbf{w}_{1}^{2}\left(\mathbb{Q}_{1}\left(\mathbf{w}_{1}, \varsigma\right)\right)_{\mathbf{w}_{1} \mathbf{w}_{1}}\right.\right. \\
& \left.\left.-0.06 \mathbf{w}_{1}\left(Q_{1}\left(\mathbf{w}_{1}, \varsigma\right)\right)_{\mathbf{w}_{1}}-0.06 Q_{1}\right]\right] \\
& =\left[-0.0036 \mathbf{w}_{1}+0.0036 \max \left(\mathbf{w}_{1}-25 \exp (-0.06), 0\right)\right] \frac{\varsigma^{29}}{\Gamma(2 \vartheta+1)}, \\
& \mathbb{Q}_{3}\left(\mathbf{w}_{1}, \varsigma\right)=\mathbb{J}^{-1}\left[\frac { 1 } { \psi ^ { 9 } ( \varsigma ) } \mathbb { J } \left[-0.08\left(2+\sin \mathbf{w}_{1}\right)^{2} \mathbf{w}_{1}^{2}\left(\mathscr{Q}_{2}\left(\mathbf{w}_{1}, \varsigma\right)\right)_{\mathbf{w}_{1} \mathbf{w}_{1}}\right.\right. \\
& \left.\left.-0.06 \mathbf{w}_{1}\left(Q_{2}\left(\mathbf{w}_{1}, \varsigma\right)\right)_{\mathbf{w}_{1}}-0.06 Q_{2}\right]\right] \\
& =\left[-0.000216 \mathbf{w}_{1}+0.00216 \max \left(\mathbf{w}_{1}-25 \exp (-0.06), 0\right)\right] \\
& \frac{\varsigma^{39}}{\Gamma(3 \vartheta+1)} \text {, }
\end{aligned}
$$$$
\vdots
$$

The approximate solution for Example 2 is expressed as

$$
\begin{aligned}
\mathscr{Q}\left(\mathbf{w}_{1}, \varsigma\right)= & \mathbb{Q}_{0}\left(\mathbf{w}_{1}, \varsigma\right)+\mathscr{Q}_{1}\left(\mathbf{w}_{1}, \varsigma\right)+\mathscr{Q}_{2}\left(\mathbf{w}_{1}, \varsigma\right)+Q_{3}\left(\mathbf{w}_{1}, \varsigma\right) \\
& +\cdots,=\max \left(\mathbf{w}_{1}-25 \exp (-0.06), 0\right) \\
& +\left(\mathbf{w}_{1}-\max \left(\mathbf{w}_{1}-25 \exp (-0.06)\right)\right) \\
& \times\left[1-1-\frac{0.06 \varsigma^{9}}{\Gamma(\vartheta+1)}-\frac{0.0036 \varsigma^{29}}{\Gamma(2 \vartheta+1)}-\frac{0.000216 \varsigma^{39}}{\Gamma(3 \vartheta+1)}+\cdots\right] \\
= & \max \left(\mathbf{w}_{1}-25 \exp (-0.06), 0\right) \\
& +\left(\mathbf{w}_{1}-\max \left(\mathbf{w}_{1}-25 \exp (-0.06), 0\right)\right)\left[1-E_{\vartheta}\left(0.06(\varsigma)^{9}\right)\right] .
\end{aligned}
$$

Case 2. Now, we solve the (6) by using ABC fractional derivative operator incorporating the Jafari decomposition method.

Considering (64) and using the differentian rule of Jafari transform, we have

$$
\begin{gathered}
\frac{\psi^{9}(\varsigma) \mathbb{A}(\vartheta)}{\vartheta+(1-\vartheta) \psi^{9}(\varsigma)} \mathbb{I}\left[\mathbb{Q}\left(\mathbf{w}_{1}, \varsigma\right)\right]=\phi(\varsigma) \sum_{q=0}^{q-1} \psi^{9-1-q}(\varsigma) \mathscr{Q}^{(q)}(0) \\
+\sqrt{ }\left[-0.08\left(2+\sin \mathbf{w}_{1}\right)^{2} \mathbf{w}_{1}^{2} \frac{\partial^{2} \mathscr{Q}\left(\mathbf{w}_{1}, \varsigma\right)}{\partial \mathbf{w}_{1}^{2}}\right. \\
\left.-0.06 \mathbf{w}_{1} \frac{\partial \mathscr{Q}\left(\mathbf{w}_{1}, \varsigma\right)}{\partial \mathbf{w}_{1}}+0.06 \mathbb{Q}\left(\mathbf{w}_{1}, \varsigma\right)\right] .
\end{gathered}
$$

In view of (7), we get

$$
\begin{aligned}
\mathbb{D}\left[Q\left(\mathbf{w}_{1}, \varsigma\right)\right]= & \frac{\phi(\varsigma)}{\psi^{9}(\varsigma)} \max \left(\mathbf{w}_{1}-25 \exp (-0.06), 0\right)+\frac{\vartheta+(1-\vartheta) \psi^{9}(\varsigma)}{\psi^{9}(\varsigma) \mathbb{A}(\vartheta)} \\
& \cdot \sqrt{ }\left[-0.08\left(2+\sin \mathbf{w}_{1}\right)^{2} \mathbf{w}_{1}^{2} \frac{\partial^{2} \mathscr{Q}\left(\mathbf{w}_{1}, \varsigma\right)}{\partial \mathbf{w}_{1}^{2}}-0.06 \mathbf{w}_{1} \frac{\partial \mathscr{Q}\left(\mathbf{w}_{1}, \varsigma\right)}{\partial \mathbf{w}_{1}}\right. \\
& \left.+0.06 \mathbb{Q}\left(\mathbf{w}_{1}, \varsigma\right)\right] .
\end{aligned}
$$

Employing the inverse Jafari transform on both sides yields:

$$
\begin{aligned}
\mathscr{Q}\left(\mathbf{w}_{1}, \varsigma\right)= & \mathbb{J}^{-1}\left[\frac{\phi(\varsigma)}{\psi^{\vartheta}(\varsigma)} \max \left(\mathbf{w}_{1}-25 \exp (-0.06), 0\right)\right] \\
& +\mathbb{J}^{-1}\left[\frac { \vartheta + ( 1 - \vartheta ) \psi ^ { 9 } ( \varsigma ) } { \psi ^ { 9 } ( \varsigma ) \mathbb { A } ( \vartheta ) } \mathbb { \vartheta } \left[-0.08\left(2+\sin \mathbf{w}_{1}\right)^{2} \mathbf{w}_{1}^{2}\right.\right. \\
& \left.\left.\cdot \frac{\partial^{2} \mathscr{Q}\left(\mathbf{w}_{1}, \varsigma\right)}{\partial \mathbf{w}_{1}^{2}}-0.06 \mathbf{w}_{1} \frac{\partial \mathscr{Q}\left(\mathbf{w}_{1}, \varsigma\right)}{\partial \mathbf{w}_{1}}+0.06 Q\left(\mathbf{w}_{1}, \varsigma\right)\right]\right] .
\end{aligned}
$$

By the Jafari decomposition method, we find:

$$
\begin{aligned}
Q_{0}\left(\mathbf{w}_{1}, \varsigma\right) & =\mathbb{J}^{-1}\left[\frac{\phi(\varsigma)}{\psi^{9}(\varsigma)} \max \left(\mathbf{w}_{1}-25 \exp (-0.06), 0\right)\right] \\
& =\max \left(\mathbf{w}_{1}-25 \exp (-0.06), 0\right) .
\end{aligned}
$$


TABLE 1: The exact, $\mathrm{JDM}_{\mathrm{CFD}}$, and $\mathrm{JDM}_{\mathrm{ABC}}$ solutions of Example 1 at various fractional orders with varying values of $\mathbf{w}_{1}$ and $\varsigma$.

\begin{tabular}{|c|c|c|c|c|c|c|c|}
\hline $\mathbf{w}_{1}$ & $\varsigma$ & $\vartheta=0.7$ & $\vartheta=0.8$ & $\vartheta=0.9$ & $\vartheta=1\left(\mathrm{JDM}_{\mathrm{CFD}}\right)$ & $\vartheta=1\left(\mathrm{JDM}_{\mathrm{ABC}}\right)$ & Exact \\
\hline \multirow{5}{*}{0.2} & 00.1 & 0.5038479597 & 0.4840895398 & 0.4674596860 & 0.4538001114 & 0.4538001114 & 0.4537994222 \\
\hline & 00.2 & 0.6142259772 & 0.5911676480 & 0.5697105760 & 0.5502060671 & 0.5502060671 & 0.5501940469 \\
\hline & 00.3 & 0.6585618567 & 0.6371931313 & 0.6161005539 & 0.5956319295 & 0.5956319295 & 0.5955719039 \\
\hline & 00.4 & 0.6956692898 & 0.6956692898 & 0.6576549272 & 0.6380690943 & 0.6380690943 & 0.6378819374 \\
\hline & 00.5 & 0.7281682904 & 0.7121411619 & 0.6954314260 & 0.6777823827 & 0.6777823827 & 0.6773315511 \\
\hline \multirow{5}{*}{0.4} & 00.1 & 0.6801236061 & 0.6534525291 & 0.6310045745 & 0.6125660773 & 0.6125660773 & 0.6125651470 \\
\hline & 00.2 & 0.7502173029 & 0.7220537959 & 0.6958460689 & 0.6720232080 & 0.6720232080 & 0.6720085265 \\
\hline & 00.3 & 0.8043692685 & 0.7782694482 & 0.7525069160 & 0.7275064817 & 0.7275064817 & 0.7274331662 \\
\hline & 00.4 & 0.8496923896 & 0.8267500270 & 0.8032615422 & 0.7793393519 & 0.7793393519 & 0.7791107578 \\
\hline & 00.5 & 0.8893867586 & 0.8698111796 & 0.8494018620 & 0.8278452719 & 0.8278452719 & 0.8272946248 \\
\hline \multirow{5}{*}{0.6} & 00.1 & 0.8307048482 & 0.7981287212 & 0.7707107275 & 0.7707107275 & 0.7707107275 & 0.7481887600 \\
\hline & 00.2 & 0.9163174827 & 0.8819184976 & 0.8499083077 & 0.8208109997 & 0.8208109997 & 0.8207930676 \\
\hline & 00.3 & 0.9824588428 & 0.9505804504 & 0.9191140225 & 0.8885784232 & 0.8885784232 & 0.8884888755 \\
\hline & 00.4 & 1.037816628 & 1.009794763 & 0.9811058630 & 0.9518872337 & 0.9518872337 & 0.9516080283 \\
\hline & 00.5 & 1.086299440 & 1.062389774 & 1.037461777 & 1.011132498 & 1.011132498 & 1.010459936 \\
\hline \multirow{5}{*}{0.8} & 00.1 & 1.014625193 & 0.9748366216 & 0.9413482084 & 0.9138412031 & 0.9138412031 & 0.9138398152 \\
\hline & 00.2 & 1.119192701 & 1.077177686 & 1.038080351 & 1.002540819 & 1.002540819 & 1.002518917 \\
\hline & 00.3 & 1.199977940 & 1.161041584 & 1.122608402 & 1.085312137 & 1.085312137 & 1.085202763 \\
\hline & 00.4 & 1.267592092 & 1.233366109 & 1.198325407 & 1.162637693 & 1.162637693 & 1.162296670 \\
\hline & 00.5 & 1.326809132 & 1.297605800 & 1.267158676 & 1.235000022 & 1.235000022 & 1.234178553 \\
\hline \multirow{5}{*}{1.0} & 00.1 & 1.239266009 & 1.190668138 & 1.149765298 & 1.116168166 & 1.116168166 & 1.116166471 \\
\hline & 00.2 & 1.366985052 & 1.315667796 & 1.267914204 & 1.224506122 & 1.224506122 & 1.224479370 \\
\hline & 00.3 & 1.465656366 & 1.418099393 & 1.371156999 & 1.325603238 & 1.325603238 & 1.325469648 \\
\hline & 00.4 & 1.548240477 & 1.506436767 & 1.463637957 & 1.420048885 & 1.420048885 & 1.419632359 \\
\hline & 00.5 & 1.620568333 & 1.584899303 & 1.547711101 & 1.508432433 & 1.508432433 & 1.507429089 \\
\hline
\end{tabular}

Here, we surmise that the unknown function $Q\left(\mathbf{w}_{1}, \varsigma\right)$ can be written by an infinite series of the form:

$$
\begin{aligned}
& \mathcal{Q}\left(\mathbf{w}_{1}, \varsigma\right)=\sum_{q=0}^{\infty} \widehat{Q}_{q}\left(\mathbf{w}_{1}, \varsigma\right) \\
& \sum_{q=0}^{\infty} \mathcal{Q}_{q+1}\left(\mathbf{w}_{1}, \varsigma\right)=\mathbb{J}^{-1}\left[\frac { \vartheta + ( 1 - \vartheta ) \psi ^ { 9 } ( \varsigma ) } { \psi ^ { \vartheta } ( \varsigma ) \mathbb { A } ( \vartheta ) } \mathbb { J } \left[-0.08\left(2+\sin \mathbf{w}_{1}\right)^{2} \mathbf{w}_{1}^{2} \sum_{q=0}^{\infty}\right.\right. \\
& \cdot\left(Q\left(\mathbf{w}_{1}, \varsigma\right)\right)_{\mathbf{w}_{1} \mathbf{w}_{1}}-0.06 \mathbf{w}_{1} \sum_{q=0}^{\infty}\left(\mathscr{Q}\left(\mathbf{w}_{1}, \varsigma\right)\right)_{\mathbf{w}_{1}} \\
& \left.\left.+0.06 \sum_{q=0}^{\infty}\left(Q\left(\mathbf{w}_{1}, \varsigma\right)\right)\right]\right], q=0,1,2, \cdots \\
& \mathbb{Q}_{1}\left(\mathbf{w}_{1}, \varsigma\right)=\mathbb{J}^{-1}\left[\frac { \vartheta + ( 1 - \vartheta ) \psi ^ { 9 } ( \varsigma ) } { \psi ^ { 9 } ( \varsigma ) \mathbb { A } ( \vartheta ) } \sqrt { \vartheta } \left[-0.08\left(2+\sin \mathbf{w}_{1}\right)^{2} \mathbf{w}_{1}^{2}\left(\mathscr{Q}_{0}\left(\mathbf{w}_{1}, \varsigma\right)\right)_{\mathbf{w}_{1} \mathbf{w}_{1}}\right.\right. \\
& \left.\left.-0.06 \mathbf{w}_{1}\left(\mathcal{Q}_{0}\left(\mathbf{w}_{1}, \varsigma\right)\right)_{\mathbf{w}_{1}}-0.06 Q_{0}\right]\right] \\
& =\frac{\left[-0.06 \mathbf{w}_{1}+0.06 \max \left(\mathbf{w}_{1}-25 \exp (-0.06), 0\right)\right]}{\mathbb{A}(\vartheta)} \\
& \cdot\left[\vartheta \frac{\varsigma^{\vartheta}}{\Gamma(\vartheta+1)}+(1-\vartheta)\right]
\end{aligned}
$$

$$
\begin{aligned}
\mathbb{Q}_{2}\left(\mathbf{w}_{1}, \varsigma\right)= & \mathbb{J}^{-1}\left[\frac { \vartheta + ( 1 - \vartheta ) \psi ^ { \vartheta } ( \varsigma ) } { \psi ^ { 9 } ( \varsigma ) \mathbb { A } ( \vartheta ) } \mathbb { J } \left[-0.08\left(2+\sin \mathbf{w}_{1}\right)^{2} \mathbf{w}_{1}^{2}\left(\mathbb{Q}_{1}\left(\mathbf{w}_{1}, \varsigma\right)\right)_{\mathbf{w}_{1} \mathbf{w}_{1}}\right.\right. \\
& \left.\left.-0.06 \mathbf{w}_{1}\left(\mathbb{Q}_{1}\left(\mathbf{w}_{1}, \varsigma\right)\right)_{\mathbf{w}_{1}}-0.06 \mathbb{Q}_{1}\right]\right] \\
= & \frac{\left[-0.0036 \mathbf{w}_{1}+0.0036 \max \left(\mathbf{w}_{1}-25 \exp (-0.06), 0\right)\right]}{A^{2}(\vartheta)} \\
& \cdot\left[\vartheta^{2} \frac{\varsigma^{2 \vartheta}}{\Gamma(2 \vartheta+1)}+2 \vartheta(1-\vartheta) \frac{\varsigma^{9}}{\Gamma(\vartheta+1)}+(1-\vartheta)^{2}\right]
\end{aligned}
$$$$
\mathbb{Q}_{3}\left(\mathbf{w}_{1}, \varsigma\right)=\mathbb{J}^{-1}\left[\frac { \vartheta + ( 1 - \vartheta ) \psi ^ { 9 } ( \varsigma ) } { \psi ^ { 9 } ( \varsigma ) \mathbb { A } ( \vartheta ) } \rrbracket \left[-0.08\left(2+\sin \mathbf{w}_{1}\right)^{2} \mathbf{w}_{1}^{2}\left(\mathbb{Q}_{2}\left(\mathbf{w}_{1}, \varsigma\right)\right)_{\mathbf{w}_{1} \mathbf{w}_{1}}\right.\right.
$$$$
\left.\left.-0.06 \mathbf{w}_{1}\left(Q_{2}\left(\mathbf{w}_{1}, \varsigma\right)\right)_{\mathbf{w}_{1}}-0.06 Q_{2}\right]\right]
$$$$
=-\frac{\left[-0.000216 \mathbf{w}_{1}+0.00216 \max \left(\mathbf{w}_{1}-25 \exp (-0.06), 0\right)\right]}{A^{3}(9)}
$$$$
\times\left[\vartheta^{3} \frac{\varsigma^{39}}{\Gamma(3 \vartheta+1)}+39^{2}(1-\vartheta) \frac{\varsigma^{29}}{\Gamma(2 \vartheta+1)}+3 \vartheta(1-\vartheta)^{2}\right.
$$$$
\left.\frac{\varsigma^{9}}{\Gamma(\vartheta+1)}+(1-\vartheta)^{3}\right] \text {. }
$$$$
\vdots
$$ 
TABLE 2: The exact, JITM $\mathrm{CFD}_{\mathrm{C}}$, and JITM $_{\mathrm{ABC}}$ solutions of Example 3 at various fractional orders with varying $\mathbf{w}_{1}$ and $\varsigma$.

\begin{tabular}{|c|c|c|c|c|c|c|c|}
\hline $\mathbf{w}_{1}$ & $\varsigma$ & $\vartheta=0.75$ & $\vartheta=0.85$ & $\vartheta=0.95$ & $\vartheta=1\left(\right.$ JITM $\left._{\mathrm{CFD}}\right)$ & $\vartheta=1\left(\right.$ JITM $\left._{\mathrm{ABC}}\right)$ & Exact \\
\hline \multirow{5}{*}{00.2} & 00.6 & 0.7508677004 & 0.7372780991 & 0.7227692296 & 0.7150366162 & 0.7150366162 & 0.7141141271 \\
\hline & 00.7 & 0.7792390784 & 0.7681866657 & 0.7564519988 & 0.7500966158 & 0.7500966158 & 0.7484099736 \\
\hline & 00.8 & 0.8059972934 & 0.7971941227 & 0.7881505824 & 0.7832272030 & 0.7832272030 & 0.7803872090 \\
\hline & 00.9 & 0.8316666049 & 0.8247731367 & 0.8182127671 & 0.8146931992 & 0.8146931992 & 0.8102025856 \\
\hline & 11.0 & 0.8566680138 & 0.8513290369 & 0.8469638545 & 0.8447594256 & 0.8447594256 & 0.8380022585 \\
\hline \multirow{5}{*}{00.4} & 00.6 & 0.9171118804 & 0.9005135041 & 0.8827923308 & 0.8733476954 & 0.8733476954 & 0.8722209647 \\
\hline & 00.7 & 0.9517647598 & 0.9382653126 & 0.9239325579 & 0.9161700757 & 0.9161700757 & 0.9141100062 \\
\hline & 00.8 & 0.9844473175 & 0.9736951006 & 0.9626492954 & 0.9566358662 & 0.9566358662 & 0.9531670898 \\
\hline & 00.9 & 1.015799885 & 1.007380184 & 0.9993673307 & 0.9950685208 & 0.9950685208 & 0.9895836730 \\
\hline & 11.0 & 1.046336675 & 1.039815634 & 1.034483988 & 1.031791493 & 1.031791493 & 1.023538270 \\
\hline \multirow{5}{*}{0.6} & 00.6 & 1.120162980 & 1.099889677 & 1.078244987 & 1.066709284 & 1.066709284 & 1.065333092 \\
\hline & 00.7 & 1.162488102 & 1.145999840 & 1.128493774 & 1.119012657 & 1.119012657 & 1.116496482 \\
\hline & 00.8 & 1.202406668 & 1.189273881 & 1.175782504 & 1.168437685 & 1.168437685 & 1.164200912 \\
\hline & 00.9 & 1.240700781 & 1.230416935 & 1.220630014 & 1.215379436 & 1.215379436 & 1.208680227 \\
\hline & 11.0 & 1.277998501 & 1.270033683 & 1.263521596 & 1.260232974 & 1.260232974 & 1.250152466 \\
\hline \multirow{5}{*}{0.8} & 00.6 & 1.368170153 & 1.343408286 & 1.316971402 & 1.302881661 & 1.302881661 & 1.301200776 \\
\hline & 00.7 & 1.419866175 & 1.399727366 & 1.378345408 & 1.366765146 & 1.366765146 & 1.363691883 \\
\hline & 00.8 & 1.468622821 & 1.452582399 & 1.436103994 & 1.427133012 & 1.427133012 & 1.421958205 \\
\hline & 00.9 & 1.515395356 & 1.502834639 & 1.490880866 & 1.484467795 & 1.484467795 & 1.476285363 \\
\hline & 11.0 & 1.560950894 & 1.551222644 & 1.543268762 & 1.539252031 & 1.539252031 & 1.526939670 \\
\hline \multirow{5}{*}{1.0} & 00.6 & 1.671086799 & 1.640842586 & 1.608552502 & 1.591343254 & 1.591343254 & 1.589290217 \\
\hline & 00.7 & 1.734228462 & 1.709630866 & 1.683514884 & 1.669370718 & 1.669370718 & 1.665617027 \\
\hline & 00.8 & 1.793779965 & 1.774188148 & 1.754061379 & 1.743104197 & 1.743104197 & 1.736783674 \\
\hline & 00.9 & 1.850908068 & 1.835566372 & 1.820966001 & 1.813133059 & 1.813133059 & 1.803139015 \\
\hline & 11.0 & 1.906549727 & 1.894667615 & 1.884952723 & 1.880046676 & 1.880046676 & 1.865008325 \\
\hline
\end{tabular}

The approximate solution for Example 2 is expressed as:

$$
\begin{aligned}
\mathbb{Q}\left(\mathbf{w}_{1}, \varsigma\right)= & \mathbb{Q}_{0}\left(\mathbf{w}_{1}, \varsigma\right)+\mathbb{Q}_{1}\left(\mathbf{w}_{1}, \varsigma\right)+\mathbb{Q}_{2}\left(\mathbf{w}_{1}, \varsigma\right)+\mathbb{Q}_{3}\left(\mathbf{w}_{1}, \varsigma\right)+\cdots, \\
= & \max \left(\mathbf{w}_{1}-25 \exp (-0.06), 0\right) \\
& -\frac{0.06 \mathbf{w}_{1}-0.06 \max \left(\mathbf{w}_{1}-25 \exp (-0.06), 0\right)}{\mathbb{A}(\vartheta)} \\
& \cdot\left[\vartheta \frac{\varsigma^{\vartheta}}{\Gamma(\vartheta+1)}+\vartheta\right] \\
& -\frac{0.0036 \mathbf{w}_{1}-0.0036 \max \left(\mathbf{w}_{1}-25 \exp (-0.06), 0\right)}{\mathbb{A}^{2}(\vartheta)} \\
& \cdot\left[\vartheta^{2} \frac{\varsigma^{2 \vartheta}}{\Gamma(2 \vartheta+1)}+2 \vartheta(1-\vartheta) \frac{\varsigma^{\vartheta}}{\Gamma(\vartheta+1)}+(1-\vartheta)^{2}\right]-\cdots
\end{aligned}
$$

The integer-order solution of Example 2 can be obtained with the aid of Taylor series expansion and setting $\vartheta=1$ as follows:

$$
\begin{aligned}
\mathcal{Q}\left(\mathbf{w}_{1}, \varsigma\right)= & \max \left(\mathbf{w}_{1}-25 \exp (-0.06), 0\right) \\
& +\left(\mathbf{w}_{1}-\max \left(\mathbf{w}_{1}-25 \exp (-0.06), 0\right)\right) \\
& \cdot[1-\exp (-0.06 \varsigma)] .
\end{aligned}
$$

\subsection{Jafari Iterative Transform Method}

Example 3 (see[16]). Assume the time-fractional onedimensional BSM (4) subject to IC (5).

Case 1. First, we surmise the Caputo fractional derivative operator for (4) with the new iterative transform method via the Jafari transform.

Implementing the Jafari transform to (4) with IC (5), we have:

$$
\begin{aligned}
\psi^{9}(\varsigma) \mathbb{I}\left[\mathscr{Q}\left(\mathbf{w}_{1}, \varsigma\right)\right]= & \phi(\varsigma) \sum_{q=0}^{q-1} \psi^{9-1-q}(\varsigma) \mathscr{Q}^{(q)}(0) \\
& +\sqrt{[}\left[\frac{\partial^{2} \mathscr{Q}\left(\mathbf{w}_{1}, \varsigma\right)}{\partial \mathbf{w}_{1}^{2}}+(\eta-1) \frac{\partial \mathscr{Q}\left(\mathbf{w}_{1}, \varsigma\right)}{\partial \mathbf{w}_{1}}-\eta \mathscr{Q}\left(\mathbf{w}_{1}, \varsigma\right)\right] .
\end{aligned}
$$

Correspondingly, we get

$$
\begin{aligned}
& \mathbb{J}\left[Q\left(\mathbf{w}_{1}, \varsigma\right)\right]=\frac{\phi(\varsigma)}{\psi^{9}(\varsigma)} \max \left(\exp \left(\mathbf{w}_{1}\right)-1,0\right)+\frac{1}{\psi^{9}(\varsigma)} \\
& \cdot \sqrt{ }\left[\frac{\partial^{2} \mathscr{Q}\left(\mathbf{w}_{1}, \varsigma\right)}{\partial \mathbf{w}_{1}^{2}}+(\eta-1) \frac{\partial \mathscr{Q}\left(\mathbf{w}_{1}, \varsigma\right)}{\partial \mathbf{w}_{1}}-\eta \mathbb{Q}\left(\mathbf{w}_{1}, \varsigma\right)\right] \text {. }
\end{aligned}
$$


TABLE 3: The comparsion analysis among HPM [14], JDM $\mathrm{CFD}_{\mathrm{C}}$, and $\mathrm{JDM}_{\mathrm{ABC}}$ of Example 1 for approximated results of $Q\left(\mathbf{w}_{1}, \varsigma\right)$ and absolute error $E_{\text {abs }}=\left\|E^{\text {exact }}-E^{\text {approx }}\right\|$ at $\vartheta=1$ with varying values of $\mathbf{w}_{1}$ and $\varsigma$.

\begin{tabular}{|c|c|c|c|c|}
\hline $\mathbf{w}_{1}$ & $\varsigma$ & $\|$ Exact - HPM\| & $\|$ Exact $-\mathrm{JDM}_{\mathrm{CFD}} \|$ & $\|$ Exact $-J^{-D_{A B C}} \|$ \\
\hline \multirow{5}{*}{0.2} & 0.1 & $768.9000000 \times 10^{-7}$ & $761.7000000 \times 10^{-9}$ & $761.7000000 \times 10^{-9}$ \\
\hline & 0.2 & $13.099020 \times 10^{-5}$ & $12.0202000 \times 10^{-6}$ & $12.0202000 \times 10^{-6}$ \\
\hline & 0.3 & $64.2248000 \times 10^{-5}$ & $60.0256000 \times 10^{-6}$ & $60.0256000 \times 10^{-6}$ \\
\hline & 0.4 & $189.107690 \times 10^{-5}$ & $187.1569000 \times 10^{-6}$ & $187.1569000 \times 10^{-6}$ \\
\hline & 0.5 & $455.4455000 \times 10^{-5}$ & $450.8316000 \times 10^{-6}$ & $450.8316000 \times 10^{-6}$ \\
\hline \multirow{5}{*}{0.4} & 0.1 & $933.050000 \times 10^{-8}$ & $930.3000000 \times 10^{-9}$ & $930.3000000 \times 10^{-9}$ \\
\hline & 0.2 & $15.7634000 \times 10^{-5}$ & $14.6815000 \times 10^{-6}$ & $14.6815000 \times 10^{-6}$ \\
\hline & 0.3 & $75.9988000 \times 10^{-5}$ & $73.3155000 \times 10^{-6}$ & $73.3155000 \times 10^{-6}$ \\
\hline & 0.4 & $230.5679000 \times 10^{-5}$ & $228.5941000 \times 10^{-6}$ & $228.5941000 \times 10^{-6}$ \\
\hline & 0.5 & $554.5621000 \times 10^{-5}$ & $550.6471000 \times 10^{-6}$ & $550.6471000 \times 10^{-6}$ \\
\hline \multirow{5}{*}{0.6} & 0.1 & $2.0098000 \times 10^{-5}$ & $1.1363000 \times 10^{-6}$ & $1.1363000 \times 10^{-6}$ \\
\hline & 0.2 & $16.4534000 \times 10^{-5}$ & $17.9321000 \times 10^{-6}$ & $17.9321000 \times 10^{-6}$ \\
\hline & 0.3 & $90.6658000 \times 10^{-5}$ & $89.5477000 \times 10^{-6}$ & $89.5477000 \times 10^{-6}$ \\
\hline & 0.4 & $220.3489000 \times 10^{-5}$ & $279.2054000 \times 10^{-6}$ & $279.2054000 \times 10^{-6}$ \\
\hline & 0.5 & $660.9890000 \times 10^{-5}$ & $672.5620000 \times 10^{-6}$ & $672.5620000 \times 10^{-6}$ \\
\hline \multirow{5}{*}{0.8} & 0.1 & $2.0094000 \times 10^{-5}$ & $1.3879000 \times 10^{-6}$ & $1.3879000 \times 10^{-6}$ \\
\hline & 0.2 & $23.0020000 \times 10^{-5}$ & $21.9020000 \times 10^{-6}$ & $21.9020000 \times 10^{-6}$ \\
\hline & 0.3 & $111.5690000 \times 10^{-5}$ & $109.3740000 \times 10^{-6}$ & $109.3740000 \times 10^{-6}$ \\
\hline & 0.8 & $345.9980000 \times 10^{-5}$ & $341.0230000 \times 10^{-6}$ & $341.0230000 \times 10^{-6}$ \\
\hline & 0.5 & $824.0000000 \times 10^{-5}$ & $821.4690000 \times 10^{-6}$ & $821.4690000 \times 10^{-6}$ \\
\hline \multirow{5}{*}{1.0} & 0.1 & $2.346789233 \times 10^{-6}$ & $1.695175303 \times 10^{-6}$ & $1.695175303 \times 10^{-6}$ \\
\hline & 0.2 & $3.700000000 \times 10^{-4}$ & $2.675000000 \times 10^{-5}$ & $2.675000000 \times 10^{-5}$ \\
\hline & 0.3 & $2.888600000 \times 10^{-3}$ & $1.336000000 \times 10^{-4}$ & $1.336000000 \times 10^{-4}$ \\
\hline & 0.4 & $5.120000000 \times 10^{-3}$ & $4.165000000 \times 10^{-4}$ & $4.165000000 \times 10^{-4}$ \\
\hline & 0.5 & $2.000000000 \times 10^{-2}$ & $1.003000000 \times 10^{-3}$ & $1.003000000 \times 10^{-3}$ \\
\hline
\end{tabular}

Employing the suggested analytical method, we obtain

$$
\begin{aligned}
& Q_{0}\left(\mathbf{w}_{1}, \varsigma\right)=\mathbb{J}^{-1}\left[\frac{\phi(\varsigma)}{\psi^{9}(\varsigma)} \max \left(\exp \left(\mathbf{w}_{1}\right)-1,0\right)\right] \\
& =\max \left(\exp \left(\mathbf{w}_{1}\right)-1,0\right) \text {, } \\
& \mathbb{Q}_{2}\left(\mathbf{w}_{1}, \varsigma\right)=\mathbb{J}^{-1}\left[\frac{1}{\psi^{9}(\varsigma)} \mathbb{J}\left[\left(\mathbb{Q}_{1}\left(\mathbf{w}_{1}, \varsigma\right)\right)_{\mathbf{w}_{1} \mathbf{w}_{1}}+(\eta-1)\left(\mathbb{Q}_{1}\left(\mathbf{w}_{1}, \varsigma\right)\right)_{\mathbf{w}_{1}}+\eta \mathscr{Q}_{1}\right]\right] \\
& =\left[-\eta^{2} \max \left(\exp \left(\mathbf{w}_{1}\right), 0\right)+\eta^{2} \max \left(\exp \left(\mathbf{w}_{1}-1\right), 0\right)\right] \frac{\varsigma^{29}}{\Gamma(2 \vartheta+1)}, \\
& \mathbb{Q}_{3}\left(\mathbf{w}_{1}, \varsigma\right)=\mathbb{J}^{-1}\left[\frac{1}{\psi^{9}(\varsigma)} \sqrt{ }\left[\left(\mathbb{Q}_{2}\left(\mathbf{w}_{1}, \varsigma\right)\right)_{\mathbf{w}_{1} \mathbf{w}_{1}}+(\eta-1)\left(\mathbb{Q}_{2}\left(\mathbf{w}_{1}, \varsigma\right)\right)_{\mathbf{w}_{1}}+\eta \mathbb{Q}_{2}\right]\right] \\
& \mathbb{Q}_{1}\left(\mathbf{w}_{1}, \varsigma\right)=\mathbb{J}^{-1}\left[\frac{1}{\psi^{9}(\varsigma)} \mathbb{J}\left[\left(\mathbb{Q}_{0}\left(\mathbf{w}_{1}, \varsigma\right)\right)_{\mathbf{w}_{1} \mathbf{w}_{1}}+(\eta-1)\left(\mathscr{Q}_{0}\left(\mathbf{w}_{1}, \varsigma\right)\right)_{\mathbf{w}_{1}}+\eta \mathscr{Q}_{0}\right]\right] \\
& =\left[-\eta^{3} \max \left(\exp \left(\mathbf{w}_{1}\right), 0\right)+\eta^{3} \max \left(\exp \left(\mathbf{w}_{1}-1\right), 0\right)\right] \frac{\varsigma^{39}}{\Gamma(39+1)} . \\
& =\left[\eta \max \left(\exp \left(\mathbf{w}_{1}\right), 0\right)-\eta \max \left(\exp \left(\mathbf{w}_{1}-1\right), 0\right)\right] \mathbb{1}^{-1}\left[\frac{\phi(\mathfrak{z})}{\psi^{9+1}(\mathfrak{g})}\right] \\
& =\left[\eta \max \left(\exp \left(\mathbf{w}_{1}\right), 0\right)-\eta \max \left(\exp \left(\mathbf{w}_{1}-1\right), 0\right)\right] \frac{\varsigma^{\vartheta}}{\Gamma(\vartheta+1)}, \\
& \vdots
\end{aligned}
$$


TABLE 4: The exact, JDM $\mathrm{JFD}_{\mathrm{CD}}$, and $\mathrm{JDM}_{\mathrm{ABC}}$ solutions of Example 2 at various fractional-orders with varying $\mathbf{w}_{1}$ and $\varsigma$.

\begin{tabular}{|c|c|c|c|c|c|c|c|}
\hline $\mathbf{w}_{1}$ & $\varsigma$ & $\vartheta=0.7$ & $\vartheta=0.8$ & $\vartheta=0.9$ & $\vartheta=1\left(J M_{\mathrm{CFD}}\right)$ & $\vartheta=1\left(J_{D M} M_{\mathrm{ABC}}\right)$ & Exact \\
\hline \multirow{5}{*}{0.2} & 0.1 & 0.0026121375 & 0.0020293922 & 0.0015639815 & 0.0011964072 & 0.0011964072 & 0.001196407 \\
\hline & 0.2 & 0.0042204353 & 0.0035172577 & 0.0029075880 & 0.0023856576 & 0.0023856576 & 0.0023856574 \\
\hline & 0.3 & 0.0055797208 & 0.0048449971 & 0.0041732529 & 0.0035677944 & 0.0035677944 & 0.003567793 \\
\hline & 0.4 & 0.0067961194 & 0.0060754949 & 0.0053880675 & 0.0047428608 & 0.0047428608 & 0.0047428580 \\
\hline & 0.5 & 0.0079145429 & 0.0072365565 & 0.0065645469 & 0.0059109000 & 0.0059109000 & 0.0059108933 \\
\hline \multirow{5}{*}{0.4} & 0.1 & 0.0052242750 & 0.0040587845 & 0.0031279630 & 0.0023928144 & 0.0023928144 & 0.0023928143 \\
\hline & 0.2 & 0.0084408706 & 0.0070345154 & 0.0058151760 & 0.0047713152 & 0.0047713152 & 0.0047713148 \\
\hline & 0.3 & 0.011159441 & 0.0096899942 & 0.0083465059 & 0.0071355888 & 0.0071355888 & 0.0071355870 \\
\hline & 0.4 & 0.013592238 & 0.012150989 & 0.010776135 & 0.0094857216 & 0.0094857216 & 0.0094857160 \\
\hline & 0.5 & 0.015829085 & 0.014473113 & 0.013129093 & 0.011821800 & 0.011821800 & 0.0118217866 \\
\hline \multirow{5}{*}{0.6} & 0.1 & 0.0078364125 & 0.0060881767 & 0.0046919445 & 0.0035892216 & 0.0035892216 & 0.0035892215 \\
\hline & 0.2 & 0.012661305 & 0.010551773 & 0.0087227641 & 0.0071569728 & 0.0071569728 & 0.0071569722 \\
\hline & 0.3 & 0.016739162 & 0.014534991 & 0.012519758 & 0.010703383 & 0.010703383 & 0.0107033805 \\
\hline & 0.4 & 0.020388358 & 0.018226484 & 0.016164202 & 0.014228582 & 0.014228582 & 0.0142285741 \\
\hline & 0.5 & 0.023743628 & 0.021709669 & 0.019693640 & 0.017732700 & 0.017732700 & 0.0177326799 \\
\hline \multirow{5}{*}{0.8} & 0.1 & 0.010448550 & 0.0081175690 & 0.0062559261 & 0.0047856288 & 0.0047856288 & 0.0047856287 \\
\hline & 0.2 & 0.016881741 & 0.014069030 & 0.011630352 & 0.0095426304 & 0.0095426304 & 0.0095426296 \\
\hline & 0.3 & 0.022318883 & 0.019379988 & 0.016693011 & 0.016693011 & 0.016693011 & 0.0142711740 \\
\hline & 0.4 & 0.027184477 & 0.024301979 & 0.021552270 & 0.018971443 & 0.018971443 & 0.0189714321 \\
\hline & 0.5 & 0.031658171 & 0.028946226 & 0.026258187 & 0.023643600 & 0.023643600 & 0.0236435732 \\
\hline \multirow{5}{*}{1.0} & 0.1 & 0.013060687 & 0.010146961 & 0.0078199076 & 0.0059820360 & 0.0059820360 & 0.005982035 \\
\hline & 0.2 & 0.021102176 & 0.017586288 & 0.014537940 & 0.011928288 & 0.011928288 & 0.011928287 \\
\hline & 0.3 & 0.027898604 & 0.024224985 & 0.020866264 & 0.017838972 & 0.017838972 & 0.017838967 \\
\hline & 0.4 & 0.033980597 & 0.030377474 & 0.026940337 & 0.023714304 & 0.023714304 & 0.023714290 \\
\hline & 0.5 & 0.039572714 & 0.036182782 & 0.032822734 & 0.029554500 & 0.029554500 & 0.029554466 \\
\hline
\end{tabular}

The series form solution is

$\mathcal{Q}\left(\mathbf{w}_{1}, \varsigma\right)=Q_{0}\left(\mathbf{w}_{1}, \varsigma\right)+\mathbb{Q}_{1}\left(\mathbf{w}_{1}, \varsigma\right)+Q_{2}\left(\mathbf{w}_{1}, \varsigma\right)+Q_{3}\left(\mathbf{w}_{1}, \varsigma\right)+\cdots$

Consequently, we have

$$
\begin{aligned}
\mathbb{Q}\left(\mathbf{w}_{1}, \varsigma\right)= & \mathbb{Q}_{0}\left(\mathbf{w}_{1}, \varsigma\right)+\mathbb{Q}_{1}\left(\mathbf{w}_{1}, \varsigma\right)+\mathbb{Q}_{2}\left(\mathbf{w}_{1}, \varsigma\right)+\mathbb{Q}_{3}\left(\mathbf{w}_{1}, \varsigma\right)+\cdots, \\
= & \max \left(\exp \left(\mathbf{w}_{1}-1\right), 0\right)\left[1-\frac{\eta \varsigma^{9}}{\Gamma(\vartheta+1)}+\frac{\eta^{2} \varsigma^{29}}{\Gamma(2 \vartheta+1)}-\frac{\eta^{3} \varsigma^{39}}{\Gamma(3 \vartheta+1)}+\cdots\right] \\
& +\max \left(\exp \left(\mathbf{w}_{1}\right), 0\right)\left[1-1+\frac{\eta \varsigma^{9}}{\Gamma(\vartheta+1)}-\frac{\eta^{2} \varsigma^{29}}{\Gamma(2 \vartheta+1)}+\frac{\eta^{3} \varsigma^{39}}{\Gamma(3 \vartheta+1)}+\cdots\right] \\
= & \max \left(\exp \left(\mathbf{w}_{1}-1\right), 0\right) E_{\vartheta}\left(-\eta(\varsigma)^{9}\right)+\max \left(\exp \left(\mathbf{w}_{1}\right), 0\right) E_{\vartheta}\left(1-\eta(\varsigma)^{9}\right) .
\end{aligned}
$$

Case 2. Now, we consider the ABC fractional derivative operator for (4) with the new iterative transform method via the Jafari transform.
Implementing the Jafari transform to (4) with IC (5), we have

$$
\begin{gathered}
\frac{\psi^{\vartheta}(\varsigma) \mathbb{A}(\vartheta)}{\vartheta+(1-\vartheta) \psi^{9}(\varsigma)} \mathbb{Q}\left[\mathcal{Q}\left(\mathbf{w}_{1}, \varsigma\right)\right]=\phi(\varsigma) \sum_{q=0}^{q-1} \psi^{9-1-q}(\varsigma) \widehat{Q}^{(q)}(0) \\
+\rrbracket\left[\frac{\partial^{2} \mathscr{Q}\left(\mathbf{w}_{1}, \varsigma\right)}{\partial \mathbf{w}_{1}^{2}}+(\eta-1) \frac{\partial Q\left(\mathbf{w}_{1}, \varsigma\right)}{\partial \mathbf{w}_{1}}-\eta Q\left(\mathbf{w}_{1}, \varsigma\right)\right] .
\end{gathered}
$$

Correspondingly, we get

$$
\begin{aligned}
\mathbb{J}\left[Q\left(\mathbf{w}_{1}, \varsigma\right)\right]= & \frac{\phi(\varsigma)}{\psi^{9}(\varsigma)} \max \left(\exp \left(\mathbf{w}_{1}\right)-1,0\right)+\frac{\vartheta+(1-\vartheta) \psi^{9}(\varsigma)}{\psi^{9}(\varsigma) \mathbb{A}(\vartheta)} \\
& \cdot \mathbb{\int}\left[\frac{\partial^{2} \mathscr{Q}\left(\mathbf{w}_{1}, \varsigma\right)}{\partial \mathbf{w}_{1}^{2}}+(\eta-1) \frac{\partial Q\left(\mathbf{w}_{1}, \varsigma\right)}{\partial \mathbf{w}_{1}}-\eta \mathbb{Q}\left(\mathbf{w}_{1}, \varsigma\right)\right] .
\end{aligned}
$$


TABLE 5: The exact, JITM $_{\mathrm{CFD}}$, and JITM $_{\mathrm{ABC}}$ solutions of Example 4 at various fractional orders with varying $\mathbf{w}_{1}$ and $\varsigma$.

\begin{tabular}{|c|c|c|c|c|c|c|c|}
\hline $\mathbf{w}_{1}$ & $\varsigma$ & $\vartheta=0.75$ & $\vartheta=0.85$ & $\vartheta=0.97$ & $\vartheta=1\left(\right.$ JITM $\left._{\mathrm{CFD}}\right)$ & $\vartheta=1\left(\right.$ JITM $\left._{\mathrm{ABC}}\right)$ & Exact \\
\hline \multirow{5}{*}{00.2} & 00.6 & 0.0086548678 & 0.0080282318 & 0.0073906661 & 0.0070719552 & 0.0070719552 & 0.0070719413 \\
\hline & 00.7 & 0.0096825555 & 0.0091221078 & 0.0085297821 & 0.0082260696 & 0.0082260696 & 0.0082260438 \\
\hline & 00.8 & 0.010667397 & 0.010185717 & 0.0096537610 & 0.0093732864 & 0.0093732864 & 0.0093732425 \\
\hline & 00.9 & 0.011615667 & 0.011222842 & 0.010763853 & 0.010513648 & 0.010513648 & 0.0105135787 \\
\hline & 11.0 & 0.012532107 & 0.012236387 & 0.011861042 & 0.011647200 & 0.011647200 & 0.0116470932 \\
\hline \multirow{5}{*}{00.4} & 00.6 & 0.017309735 & 0.016056463 & 0.014781332 & 0.014143910 & 0.014143910 & 0.0141438826 \\
\hline & 00.7 & 0.019365111 & 0.018244215 & 0.017059564 & 0.016452139 & 0.016452139 & 0.0164520877 \\
\hline & 00.8 & 0.021334795 & 0.020371435 & 0.019307522 & 0.018746572 & 0.018746572 & 0.0187464851 \\
\hline & 00.9 & 0.023231334 & 0.022445684 & 0.021527707 & 0.021027297 & 0.021027297 & 0.0210271574 \\
\hline & 11.0 & 0.025064214 & 0.024472774 & 0.023722084 & 0.023294400 & 0.023294400 & 0.0232941865 \\
\hline \multirow{5}{*}{0.6} & 00.6 & 0.025964603 & 0.024084695 & 0.022171998 & 0.021215865 & 0.021215865 & 0.0212158239 \\
\hline & 00.7 & 0.029047666 & 0.027366323 & 0.025589346 & 0.024678208 & 0.024678208 & 0.0246781316 \\
\hline & 00.8 & 0.032002192 & 0.030557153 & 0.028961283 & 0.028119859 & 0.028119859 & 0.0281197277 \\
\hline & 00.9 & 0.034847002 & 0.033668527 & 0.032291560 & 0.031540946 & 0.031540946 & 0.031540736 \\
\hline & 11.0 & 0.037596321 & 0.036709162 & 0.035583126 & 0.034941600 & 0.034941600 & 0.0349412798 \\
\hline \multirow{5}{*}{0.8} & 00.6 & 0.034619471 & 0.032112927 & 0.029562664 & 0.028287820 & 0.028287820 & 0.0282877652 \\
\hline & 00.7 & 0.038730222 & 0.036488431 & 0.034119128 & 0.032904278 & 0.032904278 & 0.032904175 \\
\hline & 00.8 & 0.042669590 & 0.040742871 & 0.038615044 & 0.037493145 & 0.037493145 & 0.0374929703 \\
\hline & 00.9 & 0.046462669 & 0.044891369 & 0.043055414 & 0.042054595 & 0.042054595 & 0.042054314 \\
\hline & 11.0 & 0.050128428 & 0.048945549 & 0.047444168 & 0.046588800 & 0.046588800 & 0.0465883731 \\
\hline \multirow{5}{*}{1.0} & 00.6 & 0.043274339 & 0.040141159 & 0.036953330 & 0.035359776 & 0.035359776 & 0.035359706 \\
\hline & 00.7 & 0.048412777 & 0.045610539 & 0.042648910 & 0.041130348 & 0.041130348 & 0.041130219 \\
\hline & 00.8 & 0.053336988 & 0.050928588 & 0.048268805 & 0.046866432 & 0.046866432 & 0.046866212 \\
\hline & 00.9 & 0.058078336 & 0.056114211 & 0.053819267 & 0.052568244 & 0.052568244 & 0.052567893 \\
\hline & 11.0 & 0.062660535 & 0.061181937 & 0.059305210 & 0.058236000 & 0.058236000 & 0.058235466 \\
\hline
\end{tabular}

Employing the suggested analytical method, we obtain

$$
\begin{aligned}
& \widehat{Q}_{0}\left(\mathbf{w}_{1}, \varsigma\right)=\mathbb{J}^{-1}\left[\frac{\phi(\varsigma)}{\psi^{9}(\varsigma)} \max \left(\exp \left(\mathbf{w}_{1}\right)-1,0\right)\right] \\
& =\max \left(\exp \left(\mathbf{w}_{1}\right)-1,0\right) \text {, } \\
& \mathbb{Q}_{1}\left(\mathbf{w}_{1}, \varsigma\right)=\mathbb{J}^{-1}\left[\frac{9+(1-9) \psi^{9}(\varsigma)}{\psi^{9}(\varsigma) \mathbb{A}(9)} \rrbracket\left[\left(\mathbb{Q}_{0}\left(\mathbf{w}_{1}, \varsigma\right)\right)_{\mathbf{w}_{1} \mathbf{w}_{1}}+(\eta-1)\left(\mathbb{Q}_{0}\left(\mathbf{w}_{1}, \varsigma\right)\right)_{\mathbf{w}_{1}}+\eta \mathscr{Q}_{0}\right]\right] \\
& =\frac{\left[\eta \max \left(\exp \left(\mathbf{w}_{1}\right), 0\right)-\eta \max \left(\exp \left(\mathbf{w}_{1}-1\right), 0\right)\right]}{\mathbb{A}(\vartheta)}\left[\vartheta \frac{\varsigma^{\vartheta}}{\Gamma(\vartheta+1)}+(1-\vartheta)\right], \\
& \mathbb{Q}_{2}\left(\mathbf{w}_{1}, \varsigma\right)=\rrbracket^{-1}\left[\frac{\vartheta+(1-9) \psi^{9}(\varsigma)}{\psi^{9}(\varsigma) \mathbb{A}(9)} \rrbracket\left[\left(\mathbb{Q}_{1}\left(\mathbf{w}_{1}, \varsigma\right)\right)_{\mathbf{w}_{1} \mathbf{w}_{1}}+(\eta-1)\left(\mathbb{Q}_{1}\left(\mathbf{w}_{1}, \varsigma\right)\right)_{\mathbf{w}_{1}}+\eta \mathbb{Q}_{1}\right]\right] \\
& =-\frac{\left[\eta^{2} \max \left(\exp \left(\mathbf{w}_{1}\right), 0\right)+\eta^{2} \max \left(\exp \left(\mathbf{w}_{1}-1\right), 0\right)\right]}{\mathbb{A}^{2}(\mathcal{9})} \\
& \cdot\left[\vartheta^{2} \frac{\varsigma^{2 \vartheta}}{\Gamma(2 \vartheta+1)}+2 \vartheta(1-\vartheta) \frac{\varsigma^{\vartheta}}{\Gamma(\vartheta+1)}+(1-\vartheta)^{2}\right] \text {, } \\
& \mathbb{Q}_{3}\left(\mathbf{w}_{1}, \varsigma\right)=\rrbracket^{-1}\left[\frac{\vartheta+(1-\vartheta) \psi^{9}(\varsigma)}{\psi^{9}(\varsigma) \mathbb{A}(\vartheta)} \rrbracket\left[\left(\mathbb{Q}_{2}\left(\mathbf{w}_{1}, \varsigma\right)\right)_{\mathbf{w}_{1} \mathbf{w}_{1}}+(\eta-1)\left(\mathbb{Q}_{2}\left(\mathbf{w}_{1}, \varsigma\right)\right)_{\mathbf{w}_{1}}+\eta \mathbb{Q}_{2}\right]\right] \\
& =-\frac{\left[\eta^{3} \max \left(\exp \left(\mathbf{w}_{1}\right), 0\right)+\eta^{3} \max \left(\exp \left(\mathbf{w}_{1}-1\right), 0\right)\right]}{\mathbb{A}^{3}(9)} \\
& \times\left[\vartheta^{3} \frac{\varsigma^{39}}{\Gamma(3 \vartheta+1)}+3 \vartheta^{2}(1-\vartheta) \frac{\varsigma^{29}}{\Gamma(2 \vartheta+1)}+39(1-9)^{2} \frac{\varsigma^{9}}{\Gamma(\vartheta+1)}+(1-9)^{3}\right]
\end{aligned}
$$

$\vdots$

The series form solution is

$\mathscr{Q}\left(\mathbf{w}_{1}, \varsigma\right)=\mathbb{Q}_{0}\left(\mathbf{w}_{1}, \varsigma\right)+\mathbb{Q}_{1}\left(\mathbf{w}_{1}, \varsigma\right)+\mathbb{Q}_{2}\left(\mathbf{w}_{1}, \varsigma\right)+\mathbb{Q}_{3}\left(\mathbf{w}_{1}, \varsigma\right)+\cdots$

Consequently, we have

$$
\begin{aligned}
\mathbb{Q}\left(\mathbf{w}_{1}, \varsigma\right)= & \mathbb{Q}_{0}\left(\mathbf{w}_{1}, \varsigma\right)+\mathbb{Q}_{1}\left(\mathbf{w}_{1}, \varsigma\right)+\mathbb{Q}_{2}\left(\mathbf{w}_{1}, \varsigma\right)+\mathbb{Q}_{3}\left(\mathbf{w}_{1}, \varsigma\right)+\cdots \\
= & \max \left(\exp \left(\mathbf{w}_{1}-1\right), 0\right)\left[1-\frac{\eta}{\mathbb{A}(\vartheta)}\left[\vartheta \frac{\varsigma^{\vartheta}}{\Gamma(\vartheta+1)}+\vartheta\right]\right. \\
& \left.-\frac{\eta^{2}}{\mathbb{A}^{2}(\vartheta)}\left[\vartheta^{2} \frac{\varsigma^{2 \vartheta}}{\Gamma(2 \vartheta+1)}+2 \vartheta(1-\vartheta) \frac{\varsigma^{\vartheta}}{\Gamma(\vartheta+1)}+(1-\vartheta)^{2}\right]-\cdots\right] \\
& +\max \left(\exp \left(\mathbf{w}_{1}\right), 0\right)\left[-\frac{\eta}{\mathbb{A}(\vartheta)}\left[\vartheta \frac{\varsigma^{\vartheta}}{\Gamma(\vartheta+1)}+\vartheta\right]\right. \\
& \left.-\frac{\eta^{2}}{\mathbb{A}^{2}(\vartheta)}\left[\vartheta^{2} \frac{\varsigma^{2 \vartheta}}{\Gamma(2 \vartheta+1)}+2 \vartheta(1-\vartheta) \frac{\varsigma^{\vartheta}}{\Gamma(\vartheta+1)}+(1-\vartheta)^{2}\right]-\cdots\right] .
\end{aligned}
$$

Example 4 (see [20]). Assume the time-fractional onedimensional BSM (6) subject to IC (7). 
TABLE 6: The comparsion analysis among HPM [14], JDM $\mathrm{JFD}_{\mathrm{C}}$, and $\mathrm{JDM}_{\mathrm{ABC}}$ of Example 2 for approximated results of $Q\left(\mathbf{w}_{1}, \varsigma\right)$ and absolute error $E_{\text {abs }}=\left\|E^{\text {exact }}-E^{\text {approx }}\right\|$ at $\vartheta=1$ with varying $\mathbf{w}_{1}$ and $\varsigma$.

\begin{tabular}{|c|c|c|c|c|}
\hline $\mathbf{w}_{1}$ & $\varsigma$ & $\|$ Exact - HPM\| & $\|$ Exact $-J_{\mathrm{CDD}} \|$ & $\|$ Exact $-J_{\mathrm{JDM}} \|$ \\
\hline \multirow{5}{*}{0.2} & 0.1 & $3.00450 \times 10^{-10}$ & $2.00000 \times 10^{-11}$ & $2.00000 \times 10^{-11}$ \\
\hline & 0.2 & $2.00000 \times 10^{-9}$ & $1.80000 \times 10^{-10}$ & $1.80000 \times 10^{-10}$ \\
\hline & 0.3 & $9.50000 \times 10^{-9}$ & $8.80000 \times 10^{-10}$ & $8.80000 \times 10^{-10}$ \\
\hline & 0.4 & $3.89000 \times 10^{-8}$ & $2.76000 \times 10^{-9}$ & $2.76000 \times 10^{-9}$ \\
\hline & 0.5 & $7.70000 \times 10^{-8}$ & $6.70000 \times 10^{-9}$ & $6.70000 \times 10^{-9}$ \\
\hline \multirow{5}{*}{0.4} & 0.1 & $5.780000 \times 10^{-10}$ & $4.00000 \times 10^{-11}$ & $4.00000 \times 10^{-11}$ \\
\hline & 0.2 & $4.80000 \times 10^{-9}$ & $3.60000 \times 10^{-10}$ & $3.60000 \times 10^{-10}$ \\
\hline & 0.3 & $2.98000 \times 10^{-8}$ & $1.76000 \times 10^{-9}$ & $1.76000 \times 10^{-9}$ \\
\hline & 0.4 & $6.45000 \times 10^{-8}$ & $5.52000 \times 10^{-9}$ & $5.52000 \times 10^{-9}$ \\
\hline & 0.5 & $2.47000 \times 10^{-7}$ & $1.34000 \times 10^{-8}$ & $1.34000 \times 10^{-8}$ \\
\hline \multirow{5}{*}{0.6} & 0.1 & $7.96600 \times 10^{-10}$ & $6.00000 \times 10^{-11}$ & $6.00000 \times 10^{-11}$ \\
\hline & 0.2 & $6.785000 \times 10^{-9}$ & $5.40000 \times 10^{-10}$ & $5.40000 \times 10^{-10}$ \\
\hline & 0.3 & $3.98000 \times 10^{-8}$ & $2.64000 \times 10^{-9}$ & $2.64000 \times 10^{-9}$ \\
\hline & 0.4 & $9.31000 \times 10^{-8}$ & $8.28000 \times 10^{-9}$ & $8.28000 \times 10^{-9}$ \\
\hline & 0.5 & $3.003000 \times 10^{-7}$ & $2.01000 \times 10^{-8}$ & $2.01000 \times 10^{-8}$ \\
\hline \multirow{5}{*}{0.8} & 0.1 & $9.890000 \times 10^{-10}$ & $8.00000 \times 10^{-11}$ & $8.00000 \times 10^{-11}$ \\
\hline & 0.2 & $9.80000 \times 10^{-9}$ & $7.20000 \times 10^{-10}$ & $7.20000 \times 10^{-10}$ \\
\hline & 0.3 & $4.94000 \times 10^{-8}$ & $3.52000 \times 10^{-9}$ & $3.52000 \times 10^{-9}$ \\
\hline & 0.4 & $2.89000 \times 10^{-7}$ & $1.10400 \times 10^{-8}$ & $1.10400 \times 10^{-8}$ \\
\hline & 0.5 & $3.60089 \times 10^{-7}$ & $2.68000 \times 10^{-8}$ & $2.68000 \times 10^{-8}$ \\
\hline \multirow{5}{*}{1.0} & 0.1 & $2.9900 \times 10^{-9}$ & $1.00000 \times 10^{-10}$ & $1.00000 \times 10^{-10}$ \\
\hline & 0.2 & $11.00011 \times 10^{-9}$ & $9.00000 \times 10^{-10}$ & $9.00000 \times 10^{-10}$ \\
\hline & 0.3 & $6.40000 \times 10^{-8}$ & $4.40000 \times 10^{-9}$ & $4.40000 \times 10^{-9}$ \\
\hline & 0.4 & $2.87000 \times 10^{-7}$ & $1.38000 \times 10^{-8}$ & $1.38000 \times 10^{-8}$ \\
\hline & 0.5 & $4.89000 \times 10^{-7}$ & $3.35000 \times 10^{-8}$ & $3.35000 \times 10^{-8}$ \\
\hline
\end{tabular}

Case 1. First, we surmise the Caputo fractional derivative operator for (6) with the new iterative transform method via the Jafari transform. have

Implementing the Jafari transform to (6) with IC (7), we

$$
\begin{aligned}
& \psi^{9}(\varsigma) \mathbb{J}\left[\mathscr{Q}\left(\mathbf{w}_{1}, \varsigma\right)\right]=\phi(\varsigma) \sum_{q=0}^{q-1} \psi^{9-1-q}(\varsigma) \mathscr{Q}^{(q)}(0) \\
&+\mathbb{J}\left[-0.08\left(2+\sin \mathbf{w}_{1}\right)^{2} \mathbf{w}_{1}^{2} \frac{\partial^{2} Q\left(\mathbf{w}_{1}, \varsigma\right)}{\partial \mathbf{w}_{1}^{2}}\right. \\
&\left.-0.06 \mathbf{w}_{1} \frac{\partial Q\left(\mathbf{w}_{1}, \varsigma\right)}{\partial \mathbf{w}_{1}}+0.06 Q\left(\mathbf{w}_{1}, \varsigma\right)\right] .
\end{aligned}
$$

Correspondingly, we get:

$$
\begin{aligned}
\mathbb{D}\left[Q\left(\mathbf{w}_{1}, \varsigma\right)\right]= & \frac{\phi(\varsigma)}{\psi^{9}(\varsigma)} \max \left(\mathbf{w}_{1}-25 \exp (-0.06), 0\right)+\frac{1}{\psi^{9}(\varsigma)} \\
& \cdot \mathbb{J}\left[-0.08\left(2+\sin \mathbf{w}_{1}\right)^{2} \mathbf{w}_{1}^{2} \frac{\partial^{2} \mathscr{Q}\left(\mathbf{w}_{1}, \varsigma\right)}{\partial \mathbf{w}_{1}^{2}}\right. \\
& \left.-0.06 \mathbf{w}_{1} \frac{\partial Q\left(\mathbf{w}_{1}, \varsigma\right)}{\partial \mathbf{w}_{1}}+0.06 \mathbb{Q}\left(\mathbf{w}_{1}, \varsigma\right)\right] .
\end{aligned}
$$

Employing the suggested analytical method, we obtain

$$
\begin{aligned}
\mathbb{Q}_{0}\left(\mathbf{w}_{1}, \varsigma\right) & =\mathbb{J}^{-1}\left[\frac{\phi(\varsigma)}{\psi^{9}(\varsigma)} \max \left(\mathbf{w}_{1}-25 \exp (-0.06), 0\right)\right] \\
& =\max \left(\mathbf{w}_{1}-25 \exp (-0.06), 0\right),
\end{aligned}
$$




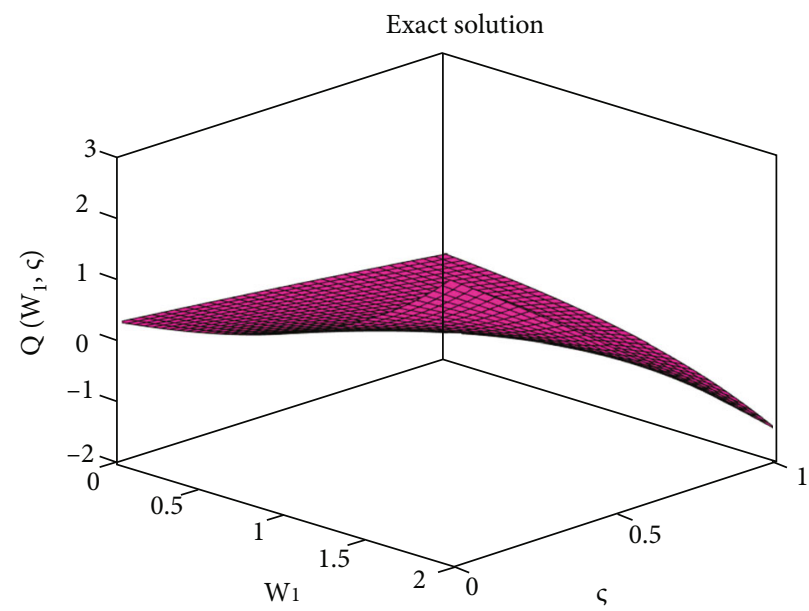

(a)

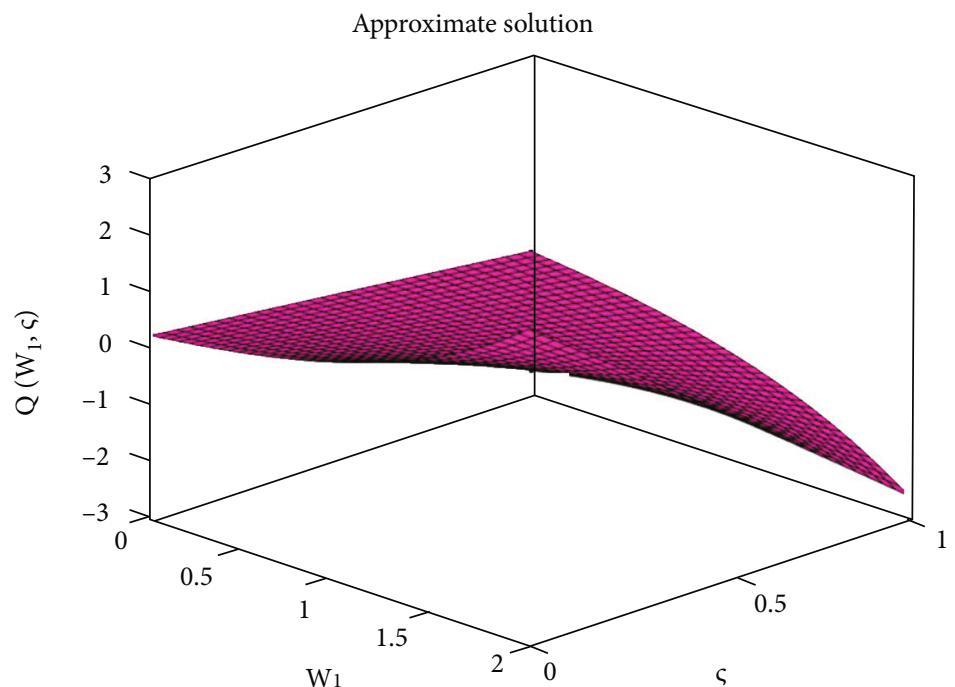

(b)

FIgURE 1: Three-dimensional illustration of the exact and approximate solution via CFD of Example 1 when $\vartheta=1$.

$$
\begin{aligned}
\mathbb{Q}_{1}\left(\mathbf{w}_{1}, \varsigma\right)= & \mathbb{J}^{-1}\left[\frac { 1 } { \psi ^ { 9 } ( \varsigma ) } \mathbb { J } \left[-0.08\left(2+\sin \mathbf{w}_{1}\right)^{2} \mathbf{w}_{1}^{2}\left(\mathbb{Q}_{0}\left(\mathbf{w}_{1}, \varsigma\right)\right)_{\mathbf{w}_{1} \mathbf{w}_{1}}\right.\right. \\
& \left.\left.-0.06 \mathbf{w}_{1}\left(\mathbb{Q}_{0}\left(\mathbf{w}_{1}, \varsigma\right)\right)_{\mathbf{w}_{1}}-0.06 Q_{0}\right]\right] \\
= & {\left[-0.06 \mathbf{w}_{1}+0.06 \max \left(\mathbf{w}_{1}-25 \exp (-0.06), 0\right)\right] \mathbb{J}^{-1}\left[\frac{\phi(\mathfrak{g})}{\psi^{9+1}(\mathfrak{g})}\right] } \\
= & {\left[-0.06 \mathbf{w}_{1}+0.06 \max \left(\mathbf{w}_{1}-25 \exp (-0.06), 0\right)\right] \frac{\varsigma^{9}}{\Gamma(\mathcal{\vartheta}+1)}, }
\end{aligned}
$$

$$
\begin{aligned}
\mathbb{Q}_{2}\left(\mathbf{w}_{1}, \varsigma\right)= & \mathbb{J}^{-1}\left[\frac { 1 } { \psi ^ { 9 } ( \varsigma ) } \mathbb { J } \left[-0.08\left(2+\sin \mathbf{w}_{1}\right)^{2} \mathbf{w}_{1}^{2}\left(\mathbb{Q}_{1}\left(\mathbf{w}_{1}, \varsigma\right)\right)_{\mathbf{w}_{1} \mathbf{w}_{1}}\right.\right. \\
& \left.\left.-0.06 \mathbf{w}_{1}\left(\mathbb{Q}_{1}\left(\mathbf{w}_{1}, \varsigma\right)\right)_{\mathbf{w}_{1}}-0.06 \mathbb{Q}_{1}\right]\right] \\
= & {\left[-0.0036 \mathbf{w}_{1}+0.0036 \max \left(\mathbf{w}_{1}-25 \exp (-0.06), 0\right)\right] \frac{\varsigma^{29}}{\Gamma(2 \vartheta+1)}, }
\end{aligned}
$$

$\vdots$

The series form solution is

$$
\mathbb{Q}\left(\mathbf{w}_{1}, \varsigma\right)=\mathbb{Q}_{0}\left(\mathbf{w}_{1}, \varsigma\right)+\mathbb{Q}_{1}\left(\mathbf{w}_{1}, \varsigma\right)+\mathbb{Q}_{2}\left(\mathbf{w}_{1}, \varsigma\right)+\mathbb{Q}_{3}\left(\mathbf{w}_{1}, \varsigma\right)+\cdots
$$




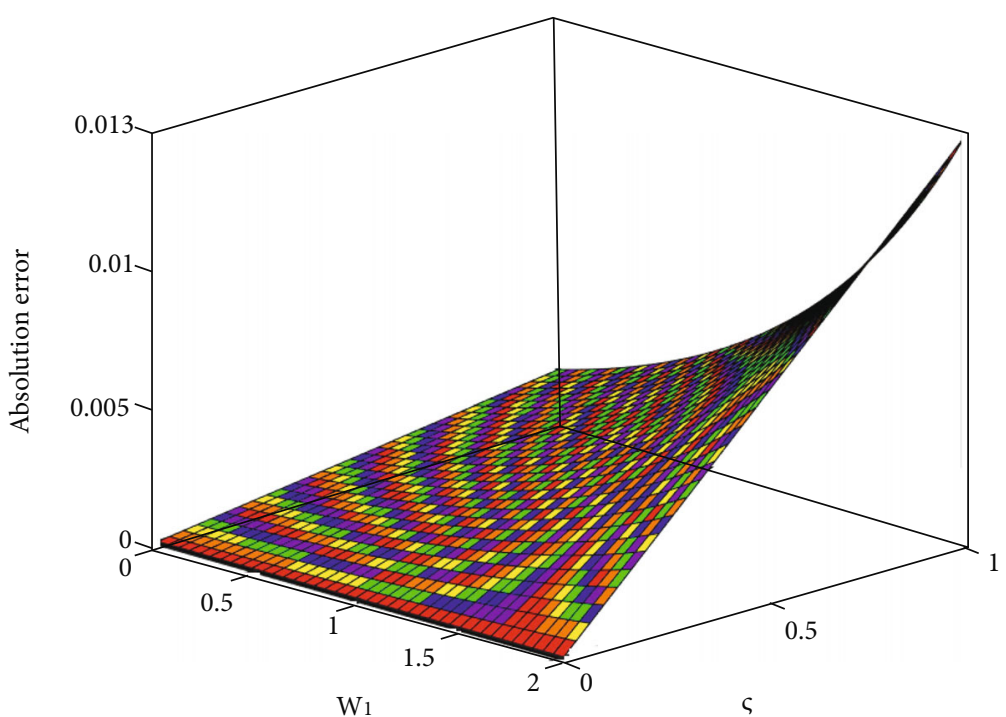

(a)

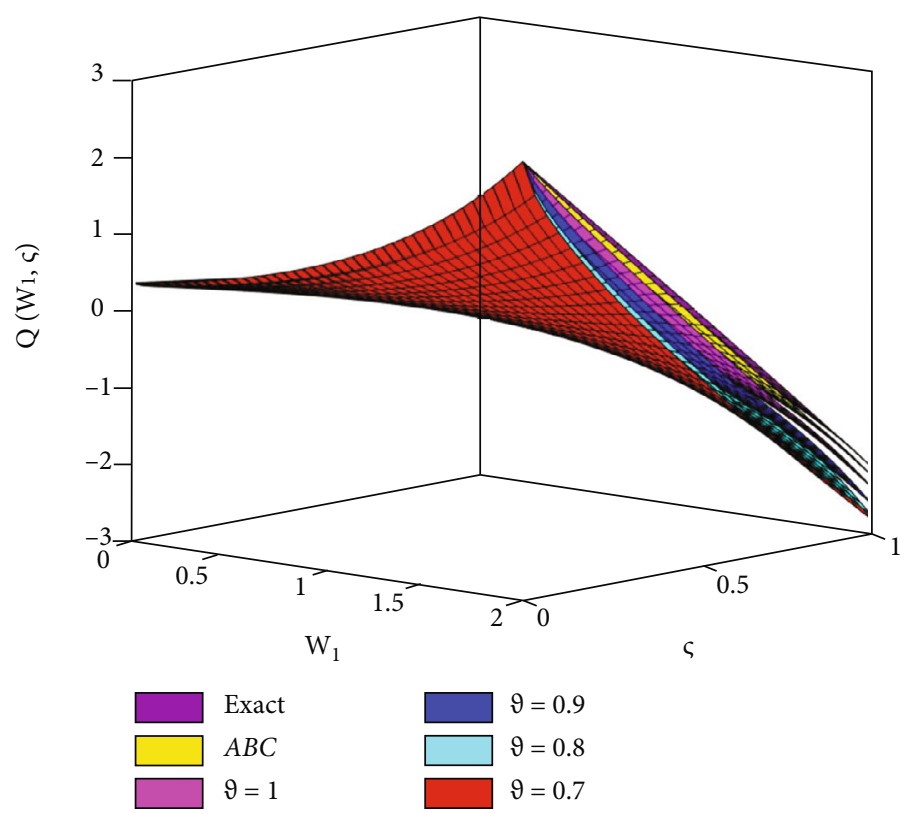

(b)

Figure 2: Three-dimensional illustration of the absolute error and multiple surface plots of Example 1 via CFD for varying fractional orders.

Consequently, we have

$$
\begin{aligned}
Q\left(\mathbf{w}_{1}, \varsigma\right)= & Q_{0}\left(\mathbf{w}_{1}, \varsigma\right)+Q_{1}\left(\mathbf{w}_{1}, \varsigma\right)+Q_{2}\left(\mathbf{w}_{1}, \varsigma\right)+Q_{3}\left(\mathbf{w}_{1}, \varsigma\right)+\cdots, \\
= & \max \left(\mathbf{w}_{1}-25 \exp (-0.06), 0\right)+\left(\mathbf{w}_{1}-\max \left(\mathbf{w}_{1}-25 \exp (-0.06)\right)\right) \\
& \times\left[1-1-\frac{0.06 \varsigma^{9}}{\Gamma(\vartheta+1)}-\frac{0.0036 \varsigma^{2 \vartheta}}{\Gamma(2 \vartheta+1)}-\frac{0.000216 \varsigma^{39}}{\Gamma(3 \vartheta+1)}+\cdots\right] \\
= & \max \left(\mathbf{w}_{1}-25 \exp (-0.06), 0\right)+\left(\mathbf{w}_{1}-\max \left(\mathbf{w}_{1}-25 \exp (-0.06)\right)\right) \\
& \cdot\left[1-E_{\vartheta}\left(0.06(\varsigma)^{\vartheta}\right)\right] .
\end{aligned}
$$

Case 2. Now, we consider the $\mathrm{ABC}$ fractional derivative operator for (6) with the new iterative transform method via the Jafari transform.
Implementing the Jafari transform to (6) with IC (7), we have

$$
\begin{aligned}
& \frac{\psi^{9}(\varsigma) \mathbb{A}(\vartheta)}{\vartheta+(1-\vartheta) \psi^{9}(\varsigma)} \mathbb{I}\left[\mathbb{Q}\left(\mathbf{w}_{1}, \varsigma\right)\right]=\phi(\varsigma) \sum_{q=0}^{q-1} \psi^{9-1-q}(\varsigma) \mathbb{Q}^{(q)}(0) \\
& +\sqrt{ }\left[-0.08\left(2+\sin \mathbf{w}_{1}\right)^{2} \mathbf{w}_{1}^{2} \frac{\partial^{2} Q\left(\mathbf{w}_{1}, \varsigma\right)}{\partial \mathbf{w}_{1}^{2}}\right. \\
& \left.-0.06 \mathbf{w}_{1} \frac{\partial \mathscr{Q}\left(\mathbf{w}_{1}, \varsigma\right)}{\partial \mathbf{w}_{1}}+0.06 \mathbb{Q}\left(\mathbf{w}_{1}, \varsigma\right)\right] \text {. }
\end{aligned}
$$

Correspondingly, we get 


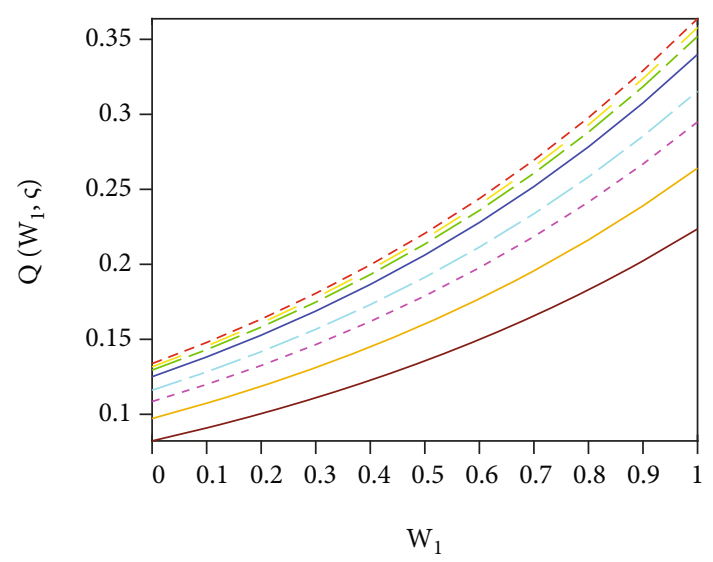

(a)

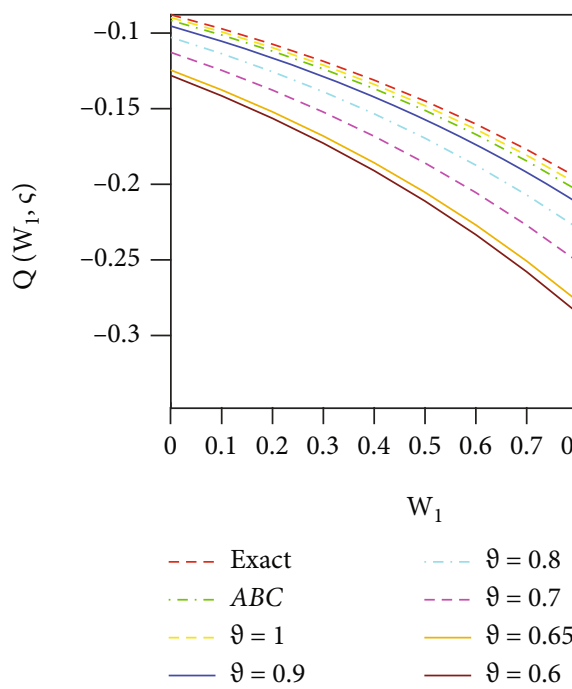

(b)

FIgURE 3: Two-dimensional illustration of the exact, approximate solution via CFD, and ABC fractional derivative operator of Example 1 with varying fractional orders at (a) $\varsigma=0.7$ and (b) $\varsigma=0.3$.

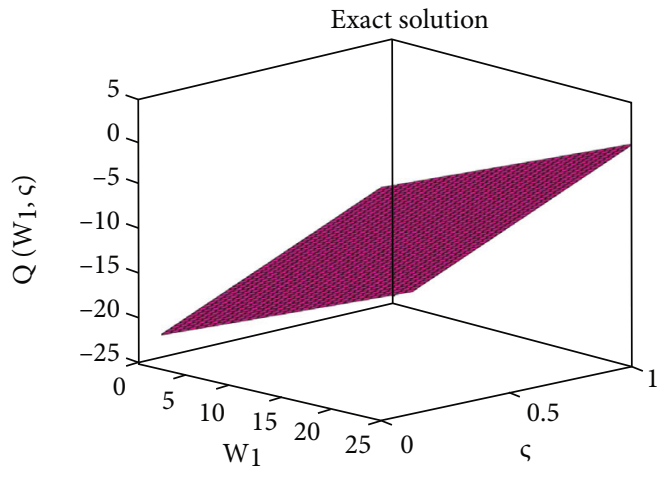

(a)

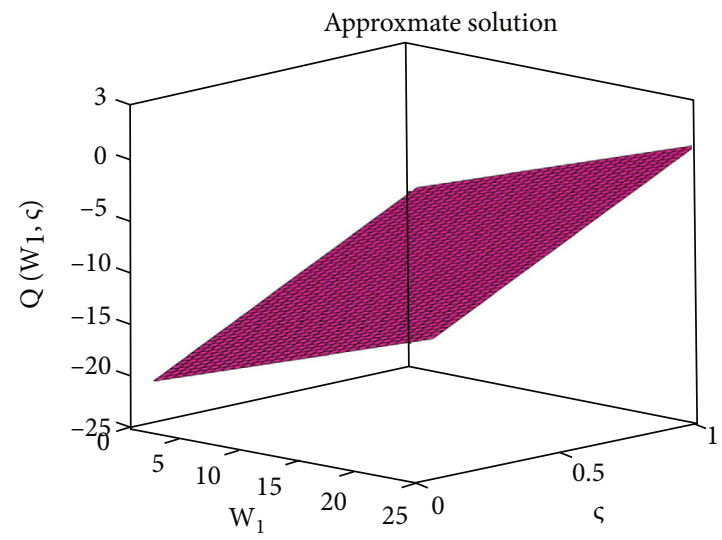

(b)

FIgURE 4: Three-dimensional illustration of the exact and approximate solution via CFD of Example 1 when $\vartheta=1$.

$$
\begin{aligned}
\mathbb{I}\left[\mathcal{Q}\left(\mathbf{w}_{1}, \varsigma\right)\right]= & \frac{\phi(\varsigma)}{\psi^{9}(\varsigma)} \max \left(\mathbf{w}_{1}-25 \exp (-0.06), 0\right)+\frac{\vartheta+(1-\vartheta) \psi^{9}(\varsigma)}{\psi^{9}(\varsigma) \mathbb{A}(\vartheta)} \\
& \mathbb{J}\left[-0.08\left(2+\sin \mathbf{w}_{1}\right)^{2} \mathbf{w}_{1}^{2} \frac{\partial^{2} \mathscr{Q}\left(\mathbf{w}_{1}, \varsigma\right)}{\partial \mathbf{w}_{1}^{2}}\right. \\
& \left.-0.06 \mathbf{w}_{1} \frac{\partial Q\left(\mathbf{w}_{1}, \varsigma\right)}{\partial \mathbf{w}_{1}}+0.06 \mathbb{Q}\left(\mathbf{w}_{1}, \varsigma\right)\right] .
\end{aligned}
$$

Employing the suggested analytical method, we obtain

$$
\begin{aligned}
\mathcal{Q}_{0}\left(\mathbf{w}_{1}, \varsigma\right) & =\mathbb{J}^{-1}\left[\frac{\phi(\varsigma)}{\psi^{9}(\varsigma)} \max \left(\mathbf{w}_{1}-25 \exp (-0.06), 0\right)\right] \\
& =\max \left(\mathbf{w}_{1}-25 \exp (-0.06), 0\right),
\end{aligned}
$$

$$
\begin{aligned}
\mathbb{Q}_{1}\left(\mathbf{w}_{1}, \varsigma\right)= & \mathbb{J}^{-1}\left[\frac { \vartheta + ( 1 - \vartheta ) \psi ^ { 9 } ( \varsigma ) } { \psi ^ { 9 } ( \varsigma ) \mathbb { A } ( \vartheta ) } \mathbb { \vartheta } \left[-0.08\left(2+\sin \mathbf{w}_{1}\right)^{2} \mathbf{w}_{1}^{2}\left(\mathbb{Q}_{0}\left(\mathbf{w}_{1}, \varsigma\right)\right)_{\mathbf{w}_{1} \mathbf{w}_{1}}\right.\right. \\
& \left.\left.-0.06 \mathbf{w}_{1}\left(\mathbb{Q}_{0}\left(\mathbf{w}_{1}, \varsigma\right)\right)_{\mathbf{w}_{1}}-0.06 \mathbb{Q}_{0}\right]\right] \\
= & \frac{\left[-0.06 \mathbf{w}_{1}+0.06 \max \left(\mathbf{w}_{1}-25 \exp (-0.06), 0\right)\right]}{\mathbb{A}(\vartheta)} \\
& \cdot\left[\vartheta \frac{\varsigma^{\vartheta}}{\Gamma(\vartheta+1)}+(1-\vartheta)\right],
\end{aligned}
$$

$$
\begin{aligned}
\mathbb{Q}_{2}\left(\mathbf{w}_{1}, \varsigma\right)= & \rrbracket^{-1}\left[\frac { \vartheta + ( 1 - \vartheta ) \psi ^ { 9 } ( \varsigma ) } { \psi ^ { 9 } ( \varsigma ) \mathbb { A } ( \vartheta ) } \rrbracket \left[-0.08\left(2+\sin \mathbf{w}_{1}\right)^{2} \mathbf{w}_{1}^{2}\left(\mathscr{Q}_{1}\left(\mathbf{w}_{1}, \varsigma\right)\right)_{\mathbf{w}_{1} \mathbf{w}_{1}}\right.\right. \\
& \left.\left.-0.06 \mathbf{w}_{1}\left(\mathscr{Q}_{1}\left(\mathbf{w}_{1}, \varsigma\right)\right)_{\mathbf{w}_{1}}-0.06 Q_{1}\right]\right] \\
= & \frac{\left[-0.0036 \mathbf{w}_{1}+0.0036 \max \left(\mathbf{w}_{1}-25 \exp (-0.06), 0\right)\right]}{\mathbb{A}^{2}(\vartheta)} \\
& \cdot\left[\vartheta^{2} \frac{\varsigma^{2 \vartheta}}{\Gamma(2 \vartheta+1)}+2 \vartheta(1-\vartheta) \frac{\varsigma^{\vartheta}}{\Gamma(\vartheta+1)}+(1-\vartheta)^{2}\right],
\end{aligned}
$$




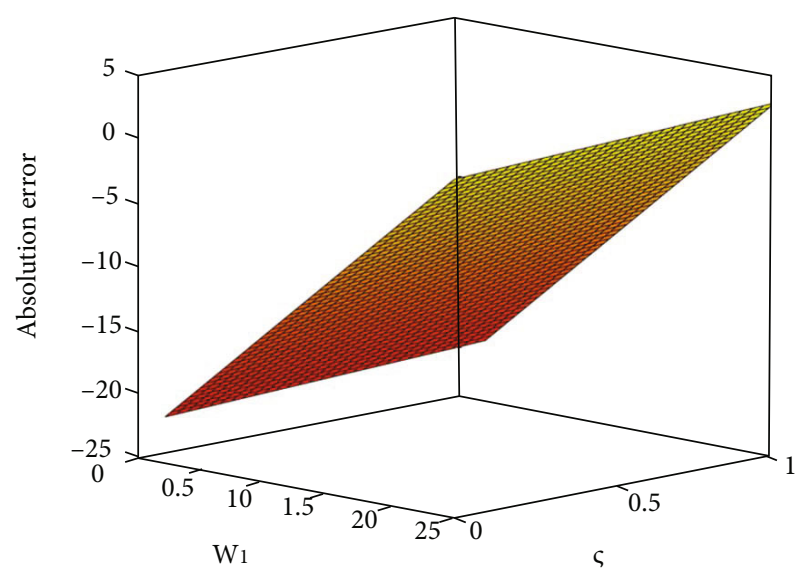

(a)

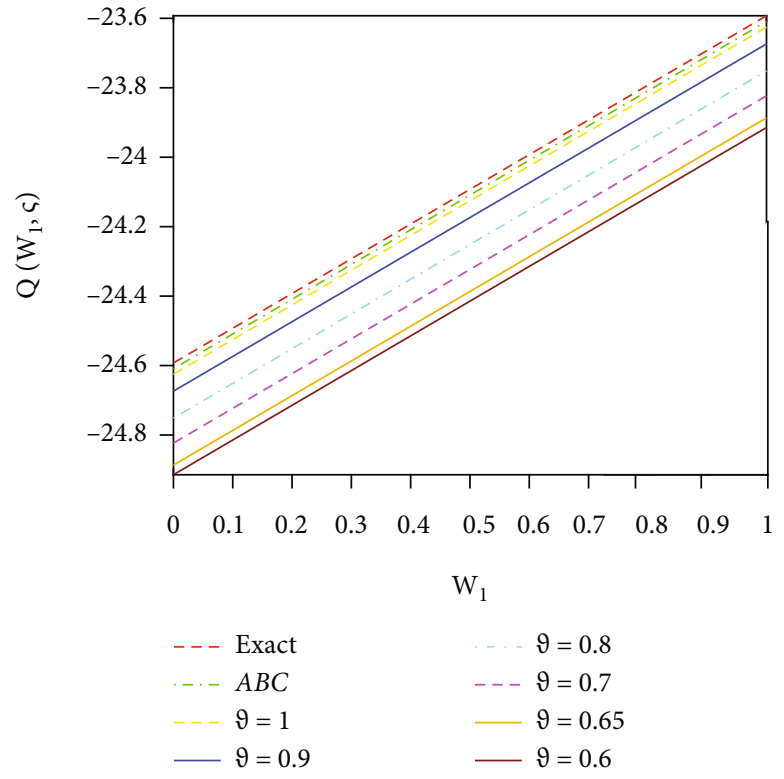

(b)

FIgURe 5: (a) Three-dimensional illustration of the absolute error plot of Example 1 when $\vartheta=1$. (b) Two-dimensional illustration of the of Example 1 for varying fractional orders when $\varsigma=0.7$.

$$
\begin{aligned}
\mathbb{Q}_{3}\left(\mathbf{w}_{1}, \varsigma\right)= & \mathbb{J}^{-1}\left[\frac { \vartheta + ( 1 - \vartheta ) \psi ^ { \vartheta } ( \varsigma ) } { \psi ^ { 9 } ( \varsigma ) \mathbb { A } ( \vartheta ) } \mathbb { J } \left[-0.08\left(2+\sin \mathbf{w}_{1}\right)^{2} \mathbf{w}_{1}^{2}\left(\mathbb{Q}_{2}\left(\mathbf{w}_{1}, \varsigma\right)\right)_{\mathbf{w}_{1} \mathbf{w}_{1}}\right.\right. \\
& \left.\left.-0.06 \mathbf{w}_{1}\left(\mathbb{Q}_{2}\left(\mathbf{w}_{1}, \varsigma\right)\right)_{\mathbf{w}_{1}}-0.06 \mathbb{Q}_{2}\right]\right] \\
= & -\frac{\left[-0.000216 \mathbf{w}_{1}+0.00216 \max \left(\mathbf{w}_{1}-25 \exp (-0.06), 0\right)\right]}{\mathbb{A}^{3}(\vartheta)} \\
& \times\left[\vartheta^{3} \frac{\varsigma^{3 \vartheta}}{\Gamma(3 \vartheta+1)}+3 \vartheta^{2}(1-\vartheta) \frac{\varsigma^{29}}{\Gamma(2 \vartheta+1)}\right. \\
& \left.+3 \vartheta(1-\vartheta)^{2} \frac{\varsigma^{9}}{\Gamma(\vartheta+1)}+(1-\vartheta)^{3}\right] .
\end{aligned}
$$$$
\vdots
$$

The series form solution is

$$
\mathscr{Q}\left(\mathbf{w}_{1}, \varsigma\right)=\mathbb{Q}_{0}\left(\mathbf{w}_{1}, \varsigma\right)+\mathbb{Q}_{1}\left(\mathbf{w}_{1}, \varsigma\right)+\mathbb{Q}_{2}\left(\mathbf{w}_{1}, \varsigma\right)+\mathbb{Q}_{3}\left(\mathbf{w}_{1}, \varsigma\right)+\cdots
$$

Consequently, we have

$$
\begin{aligned}
\mathscr{Q}\left(\mathbf{w}_{1}, \varsigma\right)= & \mathbb{Q}_{0}\left(\mathbf{w}_{1}, \varsigma\right)+\mathbb{Q}_{1}\left(\mathbf{w}_{1}, \varsigma\right)+\mathbb{Q}_{2}\left(\mathbf{w}_{1}, \varsigma\right)+\mathbb{Q}_{3}\left(\mathbf{w}_{1}, \varsigma\right) \\
& +\cdots,=\max \left(\mathbf{w}_{1}-25 \exp (-0.06), 0\right) \\
& -\frac{0.06 \mathbf{w}_{1}-0.06 \max \left(\mathbf{w}_{1}-25 \exp (-0.06), 0\right)}{\mathbb{A}(\vartheta)}\left[\vartheta \frac{\varsigma^{\vartheta}}{\Gamma(\vartheta+1)}+\vartheta\right] \\
& -\frac{0.0036 \mathbf{w}_{1}-0.0036 \max \left(\mathbf{w}_{1}-25 \exp (-0.06), 0\right)}{\mathbb{A}^{2}(\vartheta)} \\
& \cdot\left[\vartheta^{2} \frac{\varsigma^{29}}{\Gamma(2 \vartheta+1)}+2 \vartheta(1-\vartheta) \frac{\varsigma^{9}}{\Gamma(\vartheta+1)}+(1-\vartheta)^{2}\right]-\cdots
\end{aligned}
$$

\section{Results and Discussion}

Two distinctive techniques have been used to analyse the exact approximate solution of time-fractional BSM in this investigation. With MATLAB package 21, exact analytical solutions for the Black-Scholes option pricing models are presented via the $\mathrm{CFD}$ and $\mathrm{ABC}$ fractional derivative operators at any order for varying values of space and time.

For the solution in Examples 1 and 3, we conducted simulation studies for several Brownian movements with varied values of $\mathbf{w} 1$ and $\varsigma$ in Tables 1 and 2 . Table 3 exibits a numerical comparative analysis of the Homotopy perturbation approach and the Jafari decomposition method in perspective of absolute error for (4) taking into consideration both fractional derivative operators.

The findings of a simulation study for the BSM considered in Examples 2 and 4 are shown in Tables 4 and 5 . The comparative analysis of the homotopy perturbation with the projected methods is represented in Table 6. This comparison clearly illustrates that the synthesised trajectories are more powerful and effective than the existing ones.

Figure 1 depicts the behavior of the Jafari decomposition method's outcome from $Q(\mathbf{w} 1, \varsigma)$ for Example 1 . Figures 1(a) and 1(b) demonstrates the behavior of exact and approximate solutions of Black-Scholes option pricing models via the CFD operator, whereas Figures 2(a) and 2(b) depicts the absolute errors behavior and fractionalorder variation of $\mathbb{Q}\left(\mathbf{w}_{1}, \varsigma\right)$. The different fractional orders at $\vartheta=0.7,0.8,0.9,1$ behave analogously. In the same way, Figures 3(a) and 3(b) represent the two-dimensional options graphically of the obtained result $Q\left(\mathbf{w}_{1}, \varsigma\right)$ for (4) at $\varsigma=0.7$ and $\varsigma=0.3$.

Continuing in the same manner, Figures $4(\mathrm{a})$ and $4(\mathrm{~b})$ illustrates the exact -approximate consequences $Q\left(\mathbf{w}_{1}, \varsigma\right)$ 
for (1.6) by the use of Jafari decomposition method the values of the options graphically. Figures 5(a) and 5(b) show the absolute error and response of acquired data for (6) with various standard and Brownian motions of $0.6,0.65,0.7,0.8,0.9$, and 1 , respectively. Thus, we conclude that the order of the profile pictures increases as the value of the time-dependent variable increases. It is worth mentioning that the fractional order has an acceleration effect on the diffusion processes.

\section{Conclusions}

This article has investigated the more general integral transform with the Adomian decomposition method and new iterative transform method. The Caputo and $\mathrm{ABC}$ fractional derivative operators have been implemented to deal with the BSM. Several distinct solutions have been proposed with the assumption of fractional orders. Various representations have been used to understand these solutions, which have clarified the significant properties of the fractional models in consideration. Without any restrictive assumptions, discretization, or linearization, the proposed methodology locates solutions. Elegance and originality have been invoked to describe our trajectory. Contrasting proposed findings to those acquired in earlier scholarly articles demonstrate the specificality of our solutions in the European option pricing model. The strategy's powerful and successful implementation is explored and validated in order to demonstrate its applicability to additional nonlinear evolution equations that arise in banking and finance issues.

\section{Data Availability}

No data were used to support this study.

\section{Conflicts of Interest}

The authors declare that they have no competing interests.

\section{Authors' Contributions}

All authors read and approved the final manuscript.

\section{References}

[1] M. Caputo and M. Fabrizio, "A new definition of fractional derivative without singular kernel," Progress in Fractional Differentiation and Applications, vol. 73, pp. 1-13, 2015.

[2] A. Atangana and D. Baleanu, "New fractional derivatives with nonlocal and non-singular kernel: theory and application to heat transfer model," Thermal Science, vol. 20, no. 2, pp. 763-769, 2016.

[3] R. Scherer, S. L. Kalla, Y. Tang, and J. Huang, "The GrunwaldLetnikov method for fractional differential equations," Computers \& Mathematcs with Applications, vol. 62, no. 3, pp. 902-917, 2011.

[4] C. Li, D. Qian, and Y. Q. Chen, "On Riemann-Liouville and Caputo derivatives," Discrete Dynamics in Nature and Society, vol. 2011, Article ID 562494, 15 pages, 2011.
[5] V. F. Morales-Delgado, J. F. Gómez-Aguilar, H. Yépez-Martínez, D. Baleanu, R. F. Escobar-Jimenez, and V. H. OlivaresPeregrino, "Laplace homotopy analysis method for solving linear partial differential equations using a fractional derivative with and without kernel singular," Advances in Difference Equations, vol. 2016, no. 1, Article ID 164, 2016.

[6] N. A. Sheikh, F. Ali, M. Saqib et al., "Comparison and analysis of the Atangana-Baleanu and Caputo-Fabrizio fractional derivatives for generalized Casson fluid model with heat generation and chemical reaction," Results in Physics, vol. 7, pp. 789-800, 2017.

[7] A. Atangana and B. S. T. Alkahtani, "New model of groundwater flowing within a confine aquifer: application of CaputoFabrizio derivative," Arabian Journal of Geosciences, vol. 9, no. 1, 2016.

[8] D. Kumar, J. Singh, D. Baleanu, and Sushila, "Analysis of regularized long-wave equation associated with a new fractional operator with Mittag-Leffler type kernel," Physica A, vol. 492, pp. 155-167, 2018.

[9] J. Singh, D. Kumar, and D. Baleanu, "On the analysis of chemical kinetics system pertaining to a fractional derivative with Mittag-Leffler type kernel," Chaos, vol. 27, no. 10, article 103113, 2017.

[10] N. H. Luc, D. Baleanu, L. D. Long, and N. H. Can, "Reconstructing the right-hand side of a fractional subdiffusion equation from the final data," Journal of Inequalities and Applications, vol. 2020, no. 1, Article ID 53, 2020.

[11] A. I. el-Mesady, Y. S. Hamed, and A. M. Alsharif, "Jafari transformation for solving a system of ordinary differential equations with medical application," Fractal and Fractional, vol. 5, no. 3, p. 130, 2021.

[12] S. Rashid, K. T. Kubra, A. Rauf, Y. M. Chu, and Y. S. Hamed, "New numerical approach for time-fractional partial differential equations arising in physical system involving natural decomposition method," Physica Scripta, vol. 96, no. 10, p. 105204, 2021.

[13] S. Farid, R. Nawaz, Z. Shah, S. Islam, and W. Deebani, "New iterative transform method for time and space fractional $(n+1)$-dimensional heat and wave type equations," Fractals, vol. 29, no. 3, p. 2150056, 2021.

[14] A. N. Fall, S. N. Ndiaye, and N. Sene, "Black-Scholes option pricing equations described by the Caputo generalized fractional derivative," Chaos, Solitons \& Fractals, vol. 125, pp. 108-118, 2019.

[15] H. A. Zedan and E. Alaidarous, "Haar wavelet method for the system of integral equations," Abstract and Applied Analysis, vol. 2014, Article ID 418909, 9 pages, 2014.

[16] N. Özdemir and M. Yavuz, "Numerical solution of fractional Black-Scholes equation by using the multivariate padé approximation," Acta physica Polonica, vol. 132, no. 3-II, pp. 1050-1053, 2017.

[17] O. Abu Arqub, M. al-Smadi, S. Momani, and T. Hayat, "Numerical solutions of fuzzy differential equations using reproducing kernel Hilbert space method," Soft Computing, vol. 20, no. 8, pp. 3283-3302, 2016.

[18] N. Ablaoui-Lahmar, O. Belhamitib, and S. M. Bahric, "A new Legendre wavelets decomposition method for solving PDEs," Malaya Journal of Matematik, vol. 1, no. 1, pp. 72-81, 2014.

[19] S. Kumar, M. Niwas, and A. M. Wazwaz, "Lie symmetry analysis, exact analytical solutions and dynamics of solitons 
for $(2+1)$-dimensional NNV equations," Physica Scripta, vol. 95, no. 9, article 095204, 2020.

[20] Z. Cen and A. le, "A robust and accurate finite difference method for a generalized Black-Scholes equation," Journal of Computational and Applied Mathematics, vol. 235, no. 13, pp. 3728-3733, 2011.

[21] P. Wilmott, J. Dewynne, and S. Howison, Option Pricing: Mathematical Models and Computation, Oxford Financial Press, Oxford, 1993.

[22] C. Vazquez, "An upwind numerical approach for an American and European option pricing model," Applied Mathematics and Computation, vol. 97, no. 2-3, pp. 273-286, 1998.

[23] L. Meng and M. Wang, "Comparison of Black-Scholes formula with fractional Black-Scholes formula in the foreign exchange option market with changing volatility," Asia-Pacific Financial Markets, vol. 17, no. 2, pp. 99-111, 2010.

[24] Q. Fu, K. Wang, and X. Liu, "The foreign exchange option pricing based on the fractional Black-Scholes model and valuation," Price Monthly, vol. 369, pp. 68-70, 2008.

[25] M. B. Matadi and P. L. Zondi, "Invariant solutions of BlackScholes equation with Ornstein-Uhlenbeck process," Symmetry, vol. 13, no. 5, p. 847, 2021.

[26] S. Kumar, D. Kumar, and J. Singh, "Numerical computation of fractional Black-Scholes equation arising in financial market," Egyptian Journal of Basic and Applied Sciences, vol. 1, no. 3-4, pp. 177-183, 2014.

[27] M. Yavuz and N. Özdemir, "A different approach to the European option pricing model with new fractional operator," Mathematical Modelling of Natural Phenomena, vol. 13, no. 1, p. 12, 2018.

[28] S. Rashid, Z. Hammouch, H. Aydi, A. G. Ahmad, and A. M. Alsharif, "Novel computations of the time-fractional Fisher's model via generalized fractional integral operators by means of the Elzaki transform," Fractal and Fractional, vol. 5, no. 3, p. 94, 2021.

[29] S. Rashid, K. T. Kubra, and J. L. G. Guirao, "Construction of an approximate analytical solution for multi-dimensional fractional Zakharov-Kuznetsov equation via Aboodh Adomian decomposition method," Symmetry, vol. 13, no. 8, p. 1542, 2021.

[30] H. Jafari, "A new general integral transform for solving integral equations," Journal of Advanced Research, vol. 32, pp. 133-138, 2020.

[31] V. Daftardar-Gejji and H. Jafari, "An iterative method for solving nonlinear functional equations," Journal of Mathematical Analysis and Applications, vol. 316, no. 2, pp. 753-763, 2006.

[32] I. Ullah, H. Khan, and M. T. Rahim, "Numerical solutions of fifth and sixth order nonlinear boundary value problems by Daftardar Jafari method," Journal of Computational Engineering, vol. 2014, Article ID 286039, 8 pages, 2014.

[33] K. Wang and S. Liu, "Application of new iterative transform method and modified fractional homotopy analysis transform method for fractional Fornberg-Whitham equation," Journal of Nonlinear Sciences and Applications, vol. 9, no. 5, pp. 2419-2433, 2016.

[34] S. Widatalla and M. Z. Liu, "New iterative method based on Laplace decomposition algorithm," Journal of Applied Mathematics, vol. 2013, Article ID 286529, 7 pages, 2013.

[35] L. Debnath and D. Bhatta, Integral Transforms and Their Applications, CRC Press, Boca Raton, FL, USA, 2014.
[36] F. Jarad and T. Abdeljawad, "A modified Laplace transform for certain generalized fractional operators," Results in Nonlinear Analysis, vol. 1, no. 2, pp. 88-98, 2018.

[37] G. K. Watugala, "Sumudu transform: a new integral transform to solve differential equations and control engineering problems," International Journal of Mathematical Education in Science and Technology, vol. 24, no. 1, pp. 35-43, 1993.

[38] K. S. Aboodh, "The new integral transform Aboodh transform," The Global Journal of Pure and Applied Mathematics, vol. 9, pp. 35-43, 2013.

[39] S. A. P. Ahmadi, H. Hosseinzadeh, and Y. A. Cherati, "A new integral transform for solving higher order linear ordinary differential equations," Nonlinear Dynamics and Systems Theory, vol. 19, no. 2, pp. 243-252, 2019.

[40] S. A. P. Ahmadi, H. Hosseinzadeh, and A. B. Y. Cherati, "A new integral transform for solving higher order linear ordinary Laguerre and Hermite differential equations," International Journal of Applied and Computational Mathematics, vol. 5, no. $5,2019$.

[41] T. M. Elzaki, "The new integral transform Elzaki transform," Global Journal of Pure and Applied Sciences, vol. 7, no. 1, pp. 57-64, 2011.

[42] Z. H. Khan and W. A. Khan, "N-transform properties and applications," NUST Journal of Engineering Science, vol. 1, no. 1, pp. 127-133, 2008.

[43] M. M. Abdelrahim Mahgoub, "The new integral transform Mohand transform," The Advances in Theoretical and Applied Mathematics, vol. 12, no. 2, pp. 113-120, 2017.

[44] M. M. Abdelrahim Mahgoub, "The new integral transform Sawi transform," The Advances in Theoretical and Applied Mathematics, vol. 14, no. 1, pp. 81-87, 2019.

[45] H. Kamal and A. Sedeeg, "The new integral transform Kamal transform," The Advances in Theoretical and Applied Mathematics, vol. 11, no. 4, pp. 451-458, 2016.

[46] H. Kim, "On the form and properties of an integral transform with strength in integral transforms," Far East Journal of Mathematical Sciences, vol. 102, no. 11, pp. 2831-2844, 2017.

[47] H. Kim, "The intrinsic structure and properties of Laplacetyped integral transforms," Mathematical Problems in Engineering, vol. 2017, Article ID 1762729, 8 pages, 2017.

[48] M. Meddahi, H. Jafari, and M. N. Ncube, "New general integral transform via Atangana-Baleanu derivatives," Advances in Difference Equations, vol. 2021, no. 1, Article ID 385, 2021.

[49] A. Atangana and I. Koca, "Chaos in a simple nonlinear system with Atangana-Baleanu derivatives with fractional order," Chaos Solitons Fractals, vol. 89, pp. 447-454, 2016.

[50] M. Yavuz and T. Abdeljawad, "Nonlinear regularized longwave models with a new integral transformation applied to the fractional derivative with power and Mittag-Leffler kernel," Advances in Difference Equations, vol. 2020, no. 1, Article ID $367,2020$.

[51] A. Bokhari, "Application of Shehu transform to AtanganaBaleanu derivatives," The Journal of Mathematics and Computer Science, vol. 20, no. 2, pp. 101-107, 2020.

[52] M. G. Mittag-Leffler, "Sur la nouvelle fonction $\mathrm{Ea}(\mathrm{x})$," Comptes Rendus Académie des Sciences, vol. 2, p. 1003, 1903.

[53] I. L. el-Kalla, "Convergence of the Adomian method applied to a class of nonlinear integral equations," Applied Mathematics Letters, vol. 21, no. 4, pp. 372-376, 2008. 